\title{
Relaxed Inertial Fixed Point Method for Infinite \\ Family of Averaged Quasi-Nonexpansive Mapping with Applications to Sparse Signal Recovery
}

Yekini Shehu ( $\sim$ deltanougt2006@yahoo.com )

Zhejiang Normal University https://orcid.org/0000-0001-9224-7139

Qiao-Li Dong

Civil Aviation University of China

Ziyue Hu

Civil Aviation University of China

Jen-Chih Yao

China Medical University

\section{Research Article}

Keywords: averaged quasi-nonexpansive mapping, inertial step, weak con- vergence, IQ-regularization, sparse signal recovery.

Posted Date: April 26th, 2021

DOI: https://doi.org/10.21203/rs.3.rs-187524/v1

License: (c) (i) This work is licensed under a Creative Commons Attribution 4.0 International License. Read Full License

Version of Record: A version of this preprint was published at Soft Computing on October 30th, 2021. See the published version at https://doi.org/10.1007/s00500-021-06416-7. 


\title{
Relaxed Inertial Fixed Point Method for Infinite Family of Averaged Quasi-Nonexpansive Mapping with Applications to Sparse Signal Recovery
}

\author{
Yekini Shehu*, Qiao-Li Dong ${ }^{\dagger}$ Ziyue $\mathrm{Hu}^{\ddagger}$ and Jen-Chih Yao ${ }^{\S}$
}

January 20, 2021

\begin{abstract}
It is known that several optimization problems can be converted to a fixed point problem for which the underline fixed point operator is an averaged quasi-nonexpansive mapping and thus the corresponding fixed point method utilize to solve the considered optimization problem. In this paper, we consider a fixed point method involving inertial extrapolation step with relaxation parameter to obtain a common fixed point of a countable family of averaged quasi-nonexpansive mappings in real Hilbert spaces. Our results bring a unification of several versions of fixed point methods for averaged quasi-nonexpansive mappings considered in the literature and give several implications of our results. We also give some applications to monotone inclusion problem with three-operator splitting method and composite convex and non-convex relaxed inertial proximal methods to solve both convex and nonconvex reweighted $l_{Q}$ regularization for recovering a sparse signal. Finally, some numerical experiments are drawn from sparse signal recovery to illustrate our theoretical results.
\end{abstract}

Keywords: averaged quasi-nonexpansive mapping; inertial step; weak convergence; $l_{Q}$-regularization; sparse signal recovery.

2010 MSC classification: 47H05, 47J20, 47J25, 65K15, 90C25.

\section{Introduction}

Let us consider the real Hilbert space setting: $H$ represents a real Hilbert space with scalar product $\langle.,$.$\rangle and induced norm \|\cdot\|$. Let $T: H \rightarrow H$ be a nonlinear

\footnotetext{
*Department of Mathematics, Zhejiang Normal University, Jinhua, 321004, China; e-mail: yekini.shehu@unn.edu.ng

${ }^{\dagger}$ College of Science, Civil Aviation University of China, Tianjin 300300, China.; e-mail: dongql@lsec.cc.ac.cn.

${ }^{\ddagger}$ College of Science, Civil Aviation University of China, Tianjin 300300, China.; e-mail: ziyuehumath@163.com.

${ }^{\S}$ Center for General Education, China Medical University, Taichung 40402, Taiwan; e-mail: yaojc@math.nsysu.edu.tw
} 
continuous mapping, we denote the set of fixed points of $T$ by

$$
F(T):=\{x \in H \mid T x=x\} .
$$

Many fixed point methods for solving optimization problems have averaged quasinonexpansive mappings (see Section 2 for the precise definition) as the underline fixed point operator. Prominent examples for averaged quasi-nonexpansive mappings in Hilbert spaces are the projection mapping, the proximal point mapping, the resolvent operator, and several composite maps which involve at least one of these two mappings, see, e.g., [6] for more details. Several fixed point iterations have also been discussed in the literature cf. [8, 12, 13] and references therein for some relevant results in this direction.

It is known that the standard proximal point algorithm (PPA)

$$
x_{n+1}=\operatorname{prox}_{\lambda g}\left(x_{n}\right)
$$

comes from an implicit discretization of the first order steepest descent method, while IPA (inertial proximal algorithm, see [2, 4, 5])

$$
x_{n+1}=\operatorname{prox}_{\lambda g}\left(x_{n}+\theta_{n}\left(x_{n}-x_{n-1}\right)\right)
$$

is a discrete version of a second order dissipative dynamical system. This latter system is usually called 'heavy ball with friction' and may be exploited in certain situations in order to accelerate the convergence of the trajectories (see [2, 7, 22, 23, 27]). Numerical simulations are presented in [20], comparing the behavior of PPA, IPA and the gradient method. It turns out that the introduction of the term $\theta_{n}$ and the two iterates $x_{n-1}, x_{n}$ considerably improves the speed of convergence for IPA. This can be explained since the vector $x_{n}-x_{n-1}$ is acting as an impulsion term and since $\theta_{n}$ is acting as a speed regulator. Then it seems natural to consider the case when the resolvent in (IPA) is replaced by a more general self-mapping in view of constructing fast and stable algorithms for fixed points problems which can be regarded as a procedure of speeding up convergence properties of other associated methods in the literature (for example, see, [9, 10, 15, 17, 19, 29]).

In [24, Maingé studied the following method to find common fixed points of infinitely many averaged quasi-nonexpansive mappings $\left\{T_{n}\right\}: x_{0}, x_{1} \in H$,

$$
x_{n+1}=T_{n}\left(x_{n}+\theta_{n}\left(x_{n}-x_{n-1}\right)\right),
$$

where $\theta_{n} \in[0,1)$. Under suitable conditions on $\theta_{n}$ and the operators $\left\{T_{n}\right\}$, Maingé 24] proved that the method (3) generates a sequence which converges weakly to an element of $S \subset F\left(T_{n}\right)$. It can be observed that both (1) and (2) are special cases of (3).

Motivated by the results of Maingé [24], our aim in this paper is

- to establish weak convergence result of an over-relaxed inertial iterative procedure for countable families of average quasi-nonexpansive mappings in real Hilbert spaces; 
- to modify our proposed method to three-operator splitting method for monotone inclusion and give some convergence results;

- to propose relaxed inertial methods to solve composite convex and non-convex optimization problems;

- to give numerical implementations of some applications from sparse signal recovery and compare our method with some recent method in the literature.

The paper is therefore organized as follows: We give some basic results we will need later in the sequel and some illustrative example in Section 2. We propose our method in Section 3 with some discussions and give its weak convergence analysis in Section 4. Section 5 gives some applications of our results to three-operator splitting method and composite convex and non-convex optimization problems. We provide some numerical experiments in Section 6 and final remarks in Section 7.

\section{Preliminaries}

Here, we give some definitions and lemmas that would be used in the next sections.

Definition 2.1. Suppose $C$ is a nonempty, closed and convex subset of $H . P_{C}$ is called the metric projection of $H$ onto $C$ if, for any point $u \in H$, there exists a unique point $P_{C} u \in C$ such that

$$
\left\|u-P_{C} u\right\| \leq\|u-y\| \quad \forall y \in C .
$$

It is well known that $P_{C}$ is a nonexpansive mapping of $H$ onto $C$ (see, [6]). Furthermore,

$$
\left\langle x-y, P_{C} x-P_{C} y\right\rangle \geq\left\|P_{C} x-P_{C} y\right\|^{2} \quad \forall x, y \in H
$$

and

$$
P_{C} x \in C \quad \text { and } \quad\left\langle x-P_{C} x, P_{C} x-y\right\rangle \geq 0 \quad \forall y \in C .
$$

This implies that

$$
\|x-y\|^{2} \geq\left\|x-P_{C} x\right\|^{2}+\left\|y-P_{C} x\right\|^{2} \quad \forall x \in H, \forall y \in C .
$$

Definition 2.2. A mapping $A: H \rightarrow H$ is called monotone on $H$ if $\langle A x-A y, x-$ $y\rangle \geq 0$ for all $x, y \in H$; and $L$-Lipschitz continuous on $H$ if there exists a constant $L>0$ such that $\|A x-A y\| \leq L\|x-y\|$ for all $x, y \in H$.

Definition 2.3. Given an operator $A: H \rightarrow H$, we say that $A$ is $\eta$-inverse strongly monotone ( $\eta$-co-coercive), if there exists $\eta>0$ such that

$$
\langle A(x)-A(y), x-y\rangle \geq \eta\|A(x)-A(y)\|^{2}, \forall x, y \in H .
$$

An operator $A: D(A) \subset H \rightarrow 2^{H}$ is said to be monotone if

$$
\langle u-v, x-y\rangle \geq 0 \quad \forall x, y \in D(A), \quad u \in A x, v \in A y,
$$


and $A$ is maximal monotone if its graph

$$
G(A):=\{(x, y): x \in D(A), y \in A x\}
$$

is not properly contained in the graph of any other monotone operator.

Suppose that $g: H \rightarrow \mathbb{R}$ is a proper, convex and lower semicontinuous functional. The subdifferential of $g$ at $x$ is

$$
\partial g(x):=\{s \mid g(y) \geq g(x)+\langle s, y-x\rangle \forall y\} .
$$

The proximity operator prox $x_{g}$ of $g$, is defined by

$$
\operatorname{prox}_{g}(x):=\operatorname{argmin}_{y}\left\{g(y)+\frac{1}{2}\|y-x\|^{2}\right\} .
$$

From [6], $\operatorname{prox}_{g}(x)=(x+\partial g(x))^{-1}, x \in H$ and $\partial g$ is maximal monotone.

Definition 2.4. For each $n \geq 1$, let $T_{n}: H \rightarrow H$ be a nonlinear mapping and $I$ the identity mapping on $H$. We say that $I-T_{n}$ is demiclosed at the origin if $\forall\left\{\xi_{n}\right\} \subset H, \forall \xi \in H, \xi$ is a weak cluster point of $\left\{\xi_{n}\right\}$ and $\xi_{n}-T_{n} \xi_{n} \rightarrow 0$ strongly implies $\xi \in F\left(T_{n}\right)$.

Definition 2.5. For each $\beta \in(0,1)$ and $n \geq 1, T_{n}$ is $\beta$-averaged quasi-nonexpansive mappings on $H$ if

$$
\left\|T_{n} x-x^{*}\right\|^{2} \leq\left\|x-x^{*}\right\|^{2}-\frac{1-\beta}{\beta}\left\|x-T_{n} x\right\|^{2}, \quad \forall\left(x, x^{*}\right) \in H \times F\left(T_{n}\right) .
$$

Clearly one can see that that every $\beta$-averaged nonexpansive mapping on $H$ is $\beta$-averaged quasi-nonexpansive mappings on $H$. In particular, the every firmly nonexpansive mappings is $\beta$-averaged quasi-nonexpansive mappings on $H$ with $\beta=\frac{1}{2}$.

As a motivational example, we show that the projection and contraction operator is $\beta$-averaged quasi-nonexpansive mappings on $H$. The projection and contraction operator is introduced in [11, 18, to solve a variational inequality problem in Hilbert spaces and it is very effective since it involves one computation of projection onto the feasible set per iteration. A variational inequality is defined as: find $x \in C$ such that

$$
\langle A x, y-x\rangle \geq 0, \quad \forall y \in C,
$$

where $C$ is a nonempty closed and convex subset of $H$ and $A: H \rightarrow H$ is a continuous operator. Let $S O L$ denote the solution set of (8).

Example 2.6. Let $A: H \rightarrow H$ be a monotone and $L$-Lipschitz operator on a nonempty closed and convex subset $C$ and $\lambda$ be a positive number. For all $x \in H, \gamma \in$ $(0,2)$ and $\lambda_{n}>0, \forall n \geq 1$, define $y_{n}:=P_{C}\left(x-\lambda_{n} A x\right), d_{n}:=\left(x-y_{n}\right)-\lambda_{n}\left(A x-A y_{n}\right)$ and $T_{n} x:=x-\gamma \rho_{n} d_{n}$ where

$$
\rho_{n}:= \begin{cases}\frac{\left\langle x-y_{n}, d_{n}\right\rangle}{\left\|d_{n}\right\|^{2}}, & d_{n} \neq 0 \\ 0, & d_{n}=0\end{cases}
$$


Then

(i) $T_{n}$ is $\beta$ averaged quasi-nonexpansive mapping with $\beta=\frac{\gamma}{2}$. Thus, we have

$$
\left\|T_{n} x-x^{*}\right\|^{2} \leq\left\|x-x^{*}\right\|^{2}-\frac{1-\beta}{\beta}\left\|x-T_{n} x\right\|^{2}, \forall x \in H, x^{*} \in F\left(T_{n}\right), n \geq 1 .
$$

(ii) Suppose that $0<a \leq \lambda_{n} \leq b<\frac{1}{L}$. Then, for each $n \geq 1, I-T_{n}$ is demiclosed at the origin.

Proof. (i) First observe that for all $n \geq 1$,

$$
\begin{gathered}
x^{*} \in F\left(T_{n}\right) \Leftrightarrow x^{*}=T_{n} x^{*}=x^{*}-\gamma \rho_{n} d_{n} \Leftrightarrow d=0 \\
\Leftrightarrow 0=d_{n}=\left(x^{*}-y_{n}\right)-\lambda_{n}\left(A x^{*}-A y_{n}\right) \Leftrightarrow x^{*}=y_{n} \Leftrightarrow x^{*}=P_{C}\left(x^{*}-\lambda_{n} A x^{*}\right) \Leftrightarrow x^{*} \in S O L .
\end{gathered}
$$

Therefore, $F\left(T_{n}\right)=S O L$. Furthermore, by Lemma 2.7 (i), we obtain

$$
\begin{aligned}
\left\|T_{n} x-x^{*}\right\|^{2}= & \left\|\left(x-x^{*}\right)-\gamma \rho_{n} d_{n}\right\|^{2} \\
= & \left\|x-x^{*}\right\|^{2}-2 \gamma \rho_{n}\left\langle x-x^{*}, d_{n}\right\rangle \\
& +\gamma^{2} \rho_{n}^{2}\left\|d_{n}\right\|^{2} .
\end{aligned}
$$

Observe that

$$
\begin{aligned}
\left\langle x-x^{*}, d_{n}\right\rangle= & \left\langle x-y_{n}, d_{n}\right\rangle \\
& +\left\langle y-x^{*}, d_{n}\right\rangle .
\end{aligned}
$$

According to $y_{n}=P_{C}\left(x-\lambda_{n} A x\right)$ and using (5), we have (since $x^{*} \in F\left(T_{n}\right)$ )

$$
\left\langle y_{n}-x^{*}, x-y_{n}-\lambda_{n} A x\right\rangle \geq 0 .
$$

Now, we have from the monotonicity of $A$ that

$$
\left\langle A y_{n}, y_{n}-x^{*}\right\rangle \geq 0
$$

since $\left\langle A x^{*}, y_{n}-x^{*}\right\rangle \geq 0$ by the fact that $x^{*} \in F\left(T_{n}\right)$. Hence

$$
\left\langle\lambda A y_{n}, y_{n}-x^{*}\right\rangle \geq 0
$$

Summing (11) and (13), we get

$$
\left\langle y_{n}-x^{*}, x-y_{n}-\lambda_{n} A x+\lambda A y_{n}\right\rangle \geq 0
$$

Thus,

$$
\left\langle y_{n}-x^{*}, d_{n}\right\rangle \geq 0
$$

Combining (10) and (14), we get

$$
\left\langle x-x^{*}, d_{n}\right\rangle \geq\left\langle x-y_{n}, d_{n}\right\rangle .
$$

Putting (15) into (9), we get (with $\rho_{n}:=\frac{\left\langle x-y_{n}, d_{n}\right\rangle}{\left\|d_{n}\right\|^{2}}$ )

$$
\left\|T_{n} x-x^{*}\right\|^{2} \leq\left\|x-x^{*}\right\|^{2}-2 \gamma \rho_{n}\left\langle x-y_{n}, d_{n}\right\rangle
$$




$$
\begin{aligned}
& +\gamma^{2} \rho_{n}^{2}\left\|d_{n}\right\|^{2} \\
= & \left\|x-x^{*}\right\|^{2}-2 \gamma \rho_{n}\left\langle x-y_{n}, d_{n}\right\rangle \\
& +\gamma^{2} \rho_{n}\left\langle x-y_{n}, d_{n}\right\rangle \\
= & \left\|x-x^{*}\right\|^{2}-\gamma(2-\gamma) \rho_{n}\left\langle x-y_{n}, d_{n}\right\rangle .
\end{aligned}
$$

Therefore,

$$
\begin{aligned}
\rho\left\langle x-y_{n}, d_{n}\right\rangle & =\left\|\rho_{n} d_{n}\right\|^{2} \\
& =\frac{1}{\gamma^{2}}\left\|T_{n} x-x\right\|^{2} .
\end{aligned}
$$

Combining (16) and (17), we get

$$
\left\|T_{n} x-x^{*}\right\|^{2} \leq\left\|x-x^{*}\right\|^{2}-\frac{(2-\gamma)}{\gamma}\left\|T_{n} x-x\right\|^{2} .
$$

This concludes (i).

(ii) $\operatorname{Let}\left\{\xi_{n}\right\} \subset H$ such that $\xi_{n_{k}} \rightarrow \xi \in H$ and $\xi_{n}-T_{n} \xi_{n} \rightarrow 0$. We show that $\xi \in F\left(T_{n}\right)$. Now,

$$
\begin{aligned}
\left\langle\xi_{n}-y_{n}, d_{n}\right\rangle & =\left\langle\xi_{n}-y_{n},\left(\xi_{n}-y_{n}\right)-\lambda_{n}\left(A \xi_{n}-A y_{n}\right)\right\rangle \\
& =\left\|\xi_{n}-y_{n}\right\|^{2}-\lambda_{n}\left\langle\xi_{n}-y_{n}, A \xi_{n}-A y_{n}\right\rangle \\
& \geq\left\|\xi_{n}-y_{n}\right\|^{2}-\lambda_{n}\left\|\xi_{n}-y_{n}\right\|\left\|A \xi_{n}-A y_{n}\right\| \\
& \geq\left(1-\lambda_{n} L\right)\left\|\xi_{n}-y_{n}\right\|^{2} \\
& \geq(1-b L)\left\|\xi_{n}-y_{n}\right\|^{2} .
\end{aligned}
$$

Furthermore,

$$
\begin{aligned}
\left\|d_{n}\right\| & =\left\|\left(\xi_{n}-y_{n}\right)-\lambda_{n}\left(A \xi_{n}-A y_{n}\right)\right\| \\
& \leq\left\|\xi_{n}-y_{n}\right\|+\lambda_{n}\left\|A \xi_{n}-A y_{n}\right\| \\
& \leq\left(1+\lambda_{n} L\right)\left\|\xi_{n}-y_{n}\right\| .
\end{aligned}
$$

Combining (19) and (20) above, we get

$$
\begin{aligned}
\rho_{n} & =\frac{\left\langle\xi_{n}-y_{n}, d_{n}\right\rangle}{\left\|d_{n}\right\|^{2}} \\
& \geq \frac{1-\lambda_{n} L}{\left(1+\lambda_{n} L\right)^{2}} \geq \frac{1-b L}{(1+a L)^{2}} .
\end{aligned}
$$

Now, by the definition of $T_{n}$, we get

$$
\begin{aligned}
\left\langle\xi_{n}-y_{n}, d_{n}\right\rangle & =\left\|\rho_{n} d_{n}\right\|^{2} \\
\frac{1}{\rho_{n} \gamma^{2}}\left\|T_{n} \xi_{n}-\xi_{n}\right\|^{2} & \\
& \leq \frac{(1+a L)^{2}}{(1-b L)^{2} \gamma^{2}}\left\|T_{n} \xi_{n}-\xi_{n}\right\|^{2} .
\end{aligned}
$$


Also, from (19) and (22), we have

$$
\begin{aligned}
\left\|\xi_{n}-y_{n}\right\|^{2} & \leq \frac{1}{1-b L}\left\langle\xi_{n}-y_{n}, d_{n}\right\rangle \\
& \leq \frac{(1+a L)^{2}}{(1-b L)^{2} \gamma^{2}}\left\|T_{n} \xi_{n}-\xi_{n}\right\|^{2} .
\end{aligned}
$$

Since $\xi_{n}-T_{n} \xi_{n} \rightarrow 0$ by our assumption, we get from (23) that $\xi_{n}-y_{n} \rightarrow 0, n \rightarrow \infty$. Using $y_{n_{k}}=P_{C}\left(\xi_{n_{k}}-\lambda_{n_{k}} A \xi_{n_{k}}\right)$ together with (5), we have

$$
\left\langle\xi_{n_{k}}-A \xi_{n_{k}}-y_{n_{k}}, x-y_{n_{k}}\right\rangle \leq 0, \forall x \in C,
$$

which implies that

$$
\left\langle\xi_{n_{k}}-y_{n_{k}}, x-y_{n_{k}}\right\rangle \leq\left\langle A \xi_{n_{k}}, x-y_{n_{k}}\right\rangle, \forall x \in C .
$$

Also using the fact that $A$ is monotone, we have

$$
\begin{aligned}
\left\langle\xi_{n_{k}}-y_{n_{k}}, x-y_{n_{k}}\right\rangle+\left\langle A \xi_{n_{k}}, y_{n_{k}}-\xi_{n_{k}}\right\rangle & \leq\left\langle A \xi_{n_{k}}, x-\xi_{n_{k}}\right\rangle \\
& \leq\left\langle A x, x-\xi_{n_{k}}\right\rangle \quad \forall x \in C .
\end{aligned}
$$

Passing to the limit in (24) gives

$$
\langle A x, x-\xi\rangle \geq 0 \quad \forall x \in C .
$$

Therefore, $\xi \in S O L=F\left(T_{n}\right), \forall n \geq 1$.

We state these lemmas we will use in our convergence.

Lemma 2.7. The following statements hold in any real Hilbert space $H$ :

(i) $\|x+y\|^{2}=\|x\|^{2}+2\langle x, y\rangle+\|y\|^{2}$ for all $x, y \in H$;

(ii) $\|x+y\|^{2} \leq\|x\|^{2}+2\langle y, x+y\rangle$ for all $x, y \in H$

(iii) $\|\alpha x+\beta y\|^{2}=\alpha(\alpha+\beta)\|x\|^{2}+\beta(\alpha+\beta)\|y\|^{2}-\alpha \beta\|x-y\|^{2}, \quad \forall x, y \in H, \alpha, \beta \in \mathbb{R}$.

Lemma 2.8. (Maingé [23]) Let $\left\{\varphi_{n}\right\},\left\{\delta_{n}\right\}$ and $\left\{\theta_{n}\right\}$ be sequences in $[0,+\infty)$ such that

$$
\varphi_{n+1} \leq \varphi_{n}+\theta_{n}\left(\varphi_{n}-\varphi_{n-1}\right)+\delta_{n}, \quad \forall n \geq 1, \sum_{n=1}^{+\infty} \delta_{n}<+\infty,
$$

and there exists a real number $\theta$ with $0 \leq \theta_{n} \leq \theta<1$ for all $n \in \mathbb{N}$. Then the following hold:

(i) $\sum_{n=1}^{+\infty}\left[\varphi_{n}-\varphi_{n-1}\right]_{+}<+\infty$, where $[t]_{+}:=\max \{t, 0\}$;

(ii) there exists $\varphi^{*} \in[0,+\infty)$ such that $\lim _{n \rightarrow \infty} \varphi_{n}=\varphi^{*}$.

Lemma 2.9. (Opial [25]) Let $C$ be a nonempty set of $H$ and $\left\{x_{n}\right\}$ be a sequence in $H$ such that the following two conditions hold:

(i) for any $x \in C, \lim _{n \rightarrow \infty}\left\|x_{n}-x\right\|$ exists;

(ii) every sequential weak cluster point of $\left\{x_{n}\right\}$ is in $C$.

Then $\left\{x_{n}\right\}$ converges weakly to a point in $C$. 


\section{Proposed Method}

We first give the following assumptions on in order to obtain our convergence analysis in Section 4 .

Assumption 3.1. (a) For each $n \geq 1,\left\{T_{n}\right\}_{n=1}^{\infty}$ is a countable family of $\beta$-averaged quasi-nonexpansive mappings on $H$;

(b) For each $n \geq 1, S \subset F\left(T_{n}\right) \neq \emptyset$;

(c) $\forall\left\{\xi_{n}\right\} \subset H, \forall \xi \in H, \xi$ is a weak cluster point of $\left\{\xi_{n}\right\}$ and $\xi_{n}-T_{n} \xi_{n} \rightarrow 0$ strongly implies $\xi \in S$.

We next give a precise statement of our proposed iterative method.

Algorithm 3.2. (S.0) Choose $\theta \in[0,1)$, and $\alpha \in\left(0, \frac{(1-\theta)^{2}}{\beta \theta(1+\theta)+\beta(1-\theta)^{2}}\right)$. Let $x_{0}, x_{1} \in$ $H$ be given starting points. Set $n:=1$.

(S.1) Compute

$$
\left\{\begin{array}{l}
w_{n}=x_{n}+\theta\left(x_{n}-x_{n-1}\right) \\
x_{n+1}=(1-\alpha) w_{n}+\alpha T_{n} w_{n}, \quad n \geq 1
\end{array}\right.
$$

(S.2) Set $n \leftarrow n+1$, and go to (S.1).

Observe that $w_{n}=x_{n+1}$ mean that $w_{n} \in S$. In our convergence analysis, we implicitly assume that this does not happen after finitely many iterations, so that Algorithm 3.2 generates an infinite sequence satisfying, in particular, $w_{n} \neq x_{n+1}, \forall n \geq 1$.

\section{Remark 3.3.}

We recall some proposed related methods in the literature which are special cases of our proposed Algorithm 3.2 .

1. Suppose we take $\theta=0, \beta=\frac{1}{2}$ in Algorithm 3.2 and $T_{n}=T, \forall n \geq 1$. Then $\alpha \in\left(0, \frac{1}{\beta}\right)$ and Algorithm 3.2 reduces to the methods in [14, 15, 17, 19].

2. If $\alpha=1$ and $\theta=0$, then Algorithm 3.2 reduces to the method studied in [10]. Taking $\alpha=1$ and $\theta \in[0,1)$, then Algorithm 3.2 reduces to the method studied in [24].

3. Take $\theta=0$, then Algorithm 3.2 reduces to the method studied in [31].

4. If $T_{n}=T, \theta \in[0,1)$ and $\beta=\frac{1}{2}$ in Algorithm 3.2 , then we have the methods studied in [1].

5. Our Algorithm 3.2 is an over-relaxation inertial method if $\theta \in[0,1)$ is chosen such that

$$
\beta<\frac{(1-\theta)^{2}}{2 \theta^{2}-\theta+1}
$$

For example, if $\beta=\frac{1}{2}$, then $1<\frac{(1-\theta)^{2}}{\beta \theta(1+\theta)+\beta(1-\theta)^{2}}<2$ with $\theta<\frac{1}{3}$. 


\section{Convergence Analysis}

We show in this section that $\left\{x_{n}\right\}$ generated by Algorithm 3.2 converges weakly to a point in $F\left(T_{n}\right)$ under Assumptions 3.1.

Lemma 4.1. Suppose Assumptions 3.1 are fulfilled. Then $\left\{x_{n}\right\}$ generated by Algorithm 3.2 above is bounded.

Proof. Let $x^{*} \in \Gamma$. Using Algorithm 3.2, we get

$$
\begin{aligned}
\left\|x_{n+1}-x^{*}\right\|^{2}= & \left\|(1-\alpha)\left(w_{n}-x^{*}\right)+\alpha\left(T_{n} w_{n}-x^{*}\right)\right\|^{2} \\
= & (1-\alpha)\left\|w_{n}-x^{*}\right\|^{2}+\alpha\left\|T_{n} w_{n}-x^{*}\right\|^{2} \\
& -\alpha(1-\alpha)\left\|w_{n}-T_{n} w_{n}\right\|^{2},
\end{aligned}
$$

Observe that

$$
x_{n+1}=(1-\alpha) w_{n}+\alpha T_{n} w_{n}
$$

and this implies

$$
T_{n} w_{n}-w_{n}=\frac{1}{\alpha}\left(x_{n+1}-w_{n}\right), \quad \forall n .
$$

Using (27) in (26) noting that $T_{n}$ is $\beta$-averaged quasi-nonexpansive mappings, we get

$$
\begin{aligned}
\left\|x_{n+1}-x^{*}\right\|^{2} \leq & (1-\alpha)\left\|w_{n}-x^{*}\right\|^{2}+\alpha\left\|w_{n}-x^{*}\right\|^{2} \\
& -\frac{(1-\beta)}{\alpha \beta}\left\|x_{n+1}-w_{n}\right\|^{2}-\frac{(1-\alpha)}{\alpha}\left\|x_{n+1}-w_{n}\right\|^{2} \\
= & \left\|w_{n}-x^{*}\right\|^{2}-\left[\frac{(1-\beta)}{\alpha \beta}+\frac{(1-\alpha)}{\alpha}\right]\left\|x_{n+1}-w_{n}\right\|^{2} .
\end{aligned}
$$

Also, by Lemma 2.7 (iii),

$$
\begin{aligned}
\left\|w_{n}-x^{*}\right\|^{2}= & \left\|x_{n}+\theta\left(x_{n}-x_{n-1}\right)-x^{*}\right\|^{2} \\
= & \left\|(1+\theta)\left(x_{n}-x^{*}\right)-\theta\left(x_{n-1}-x^{*}\right)\right\|^{2} \\
= & (1+\theta)\left\|x_{n}-x^{*}\right\|^{2}-\theta\left\|x_{n-1}-x^{*}\right\|^{2} \\
& +\theta(1+\theta)\left\|x_{n}-x_{n-1}\right\|^{2} .
\end{aligned}
$$

Observe that

$$
\begin{aligned}
\left\|x_{n+1}-w_{n}\right\|^{2}= & \left\|\left(x_{n+1}-x_{n}\right)-\theta\left(x_{n}-x_{n-1}\right)\right\|^{2} \\
= & \left\|x_{n+1}-x_{n}\right\|^{2}+\theta^{2}\left\|x_{n}-x_{n-1}\right\|^{2} \\
& -2 \theta\left\langle x_{n+1}-x_{n}, x_{n}-x_{n-1}\right\rangle \\
\geq & (1-\theta)\left\|x_{n+1}-x_{n}\right\|^{2}+\left(\theta^{2}-\theta\right)\left\|x_{n}-x_{n-1}\right\|^{2} .
\end{aligned}
$$

Using (29) and (30) in 28) we get

$$
\begin{aligned}
\left\|x_{n+1}-x^{*}\right\|^{2} \leq & \left((1+\theta)\left\|x_{n}-x^{*}\right\|^{2}-\theta\left\|x_{n-1}-x^{*}\right\|^{2}\right. \\
& +\theta(1+\theta)\left\|x_{n}-x_{n-1}\right\|^{2}-(1-\theta)\left[\frac{(2-\gamma)}{\alpha \gamma}+\frac{(1-\alpha)}{\alpha}\right]\left\|x_{n+1}-x_{n}\right\|^{2}
\end{aligned}
$$


Thus,

$$
\begin{aligned}
\left\|x_{n+1}-x^{*}\right\|^{2}-\theta\left\|x_{n}-x^{*}\right\|^{2} \leq & \left\|x_{n}-x^{*}\right\|^{2}-\theta\left\|x_{n-1}-x^{*}\right\|^{2} \\
& +\theta\left[1+\theta-(\theta-1)\left[\frac{(1-\beta)}{\alpha \beta}+\frac{(1-\alpha)}{\alpha}\right]\right]\left\|x_{n}-x_{n-1}\right\|^{2} \\
& -(1-\theta)\left[\frac{(1-\beta)}{\alpha \beta}+\frac{(1-\alpha)}{\alpha}\right]\left\|x_{n+1}-x_{n}\right\|^{2} .
\end{aligned}
$$

Define

$\Gamma_{n}:=\left\|x_{n}-x^{*}\right\|^{2}-\theta\left\|x_{n-1}-x^{*}\right\|^{2}+\theta\left[1+\theta-(\theta-1)\left[\frac{(1-\beta)}{\alpha \beta}+\frac{(1-\alpha)}{\alpha}\right]\right]\left\|x_{n}-x_{n-1}\right\|^{2}, n \geq 1$.

Then we have by (31) that

$$
\begin{aligned}
\Gamma_{n+1}-\Gamma_{n}= & \left\|x_{n+1}-x^{*}\right\|^{2}-\theta\left\|x_{n}-x^{*}\right\|^{2} \\
& \theta\left[1+\theta-(\theta-1)\left[\frac{(1-\beta)}{\alpha \beta}+\frac{(1-\alpha)}{\alpha}\right]\right]\left\|x_{n+1}-x_{n}\right\|^{2} \\
& -\left\|x_{n}-x^{*}\right\|^{2}+\theta\left\|x_{n-1}-x^{*}\right\|^{2} \\
& -\theta\left[1+\theta-(\theta-1)\left[\frac{(1-\beta)}{\alpha \beta}+\frac{(1-\alpha)}{\alpha}\right]\right]\left\|x_{n}-x_{n-1}\right\|^{2} \\
\leq & -(1-\theta)\left[\frac{(1-\beta)}{\alpha \beta}+\frac{(1-\alpha)}{\alpha}\right]\left\|x_{n+1}-x_{n}\right\|^{2} \\
& +\theta\left[1+\theta-(\theta-1)\left[\frac{(1-\beta)}{\alpha \beta}+\frac{(1-\alpha)}{\alpha}\right]\right]\left\|x_{n+1}-x_{n}\right\|^{2} .
\end{aligned}
$$

By the condition that $\theta \in[0,1), \beta \in(0,1)$ and $\alpha \in\left(0, \frac{(1-\theta)^{2}}{\beta \theta(1+\theta)+\beta(1-\theta)^{2}}\right)$, we obtain

$$
\delta:=(1-\theta)\left[\frac{(1-\beta)}{\alpha \beta}+\frac{(1-\alpha)}{\alpha}\right]-\theta\left[1+\theta-(\theta-1)\left[\frac{(1-\beta)}{\alpha \beta}+\frac{(1-\alpha)}{\alpha}\right]\right]>0 .
$$

By (32), we have

$$
\Gamma_{n+1}-\Gamma_{n} \leq-\delta\left\|x_{n+1}-x_{n}\right\|^{2} .
$$

Therefore, $\left\{\Gamma_{n}\right\}$ is non-increasing. Similarly,

$$
\begin{aligned}
\Gamma_{n}= & \left\|x_{n}-x^{*}\right\|^{2}-\theta\left\|x_{n-1}-x^{*}\right\|^{2} \\
& +\theta\left[1+\theta-(\theta-1)\left[\frac{(1-\beta)}{\alpha \beta}+\frac{(1-\alpha)}{\alpha}\right]\right]\left\|x_{n}-x_{n-1}\right\|^{2} \\
\geq & \left\|x_{n}-x^{*}\right\|^{2}-\theta\left\|x_{n-1}-x^{*}\right\|^{2} .
\end{aligned}
$$

From (35), we have

$$
\begin{aligned}
\left\|x_{n}-x^{*}\right\|^{2} & \leq \theta\left\|x_{n-1}-x^{*}\right\|^{2}+\Gamma_{n} \\
& \leq \theta\left\|x_{n-1}-x^{*}\right\|^{2}+\Gamma_{1}
\end{aligned}
$$


Note that

$$
\begin{aligned}
& \leq \theta^{n}\left\|x_{0}-x^{*}\right\|^{2}+\left(1+\cdots+\theta^{n-1}\right) \Gamma_{1} \\
& \leq \theta^{n}\left\|x_{0}-x^{*}\right\|^{2}+\frac{\Gamma_{1}}{1-\theta} .
\end{aligned}
$$

$$
\begin{aligned}
\Gamma_{n+1}= & \left\|x_{n+1}-x^{*}\right\|^{2}-\theta\left\|x_{n}-x^{*}\right\|^{2} \\
& +\theta\left[1+\theta-(\theta-1)\left[\frac{(1-\beta)}{\alpha \beta}+\frac{(1-\alpha)}{\alpha}\right]\right]\left\|x_{n+1}-x_{n}\right\|^{2} \\
\geq & -\theta\left\|x_{n}-x^{*}\right\|^{2}
\end{aligned}
$$

and this means from (36) that

$$
\begin{aligned}
-\Gamma_{n+1} & \leq \theta\left\|x_{n}-x^{*}\right\|^{2} \\
& \vdots \\
& \leq \theta^{n+1}\left\|x_{0}-x^{*}\right\|^{2}+\frac{\theta \Gamma_{1}}{1-\theta} .
\end{aligned}
$$

By (34) and (37), we get

$$
\begin{aligned}
\delta \sum_{n=1}^{k}\left\|x_{n+1}-x_{n}\right\|^{2} & \leq \Gamma_{1}-\Gamma_{k+1} \\
& \leq \theta^{k+1}\left\|x_{0}-x^{*}\right\|^{2}+\frac{\Gamma_{1}}{1-\theta} .
\end{aligned}
$$

This implies

$$
\sum_{n=1}^{\infty}\left\|x_{n+1}-x_{n}\right\|^{2} \leq \frac{\Gamma_{1}}{\delta(1-\theta)}<+\infty .
$$

Therefore, $\lim _{n \rightarrow \infty}\left\|x_{n+1}-x_{n}\right\|=0$. Also, from 25), we get

$$
\left\|w_{n}-x_{n}\right\|=\theta\left\|x_{n}-x_{n-1}\right\| \rightarrow 0, n \rightarrow \infty .
$$

Also,

$$
\left\|x_{n+1}-w_{n}\right\| \leq\left\|w_{n}-x_{n}\right\|+\left\|x_{n+1}-x_{n}\right\| \rightarrow 0, n \rightarrow \infty .
$$

and

$$
\left\|T_{n} w_{n}-w_{n}\right\|=\frac{1}{\alpha}\left\|x_{n+1}-x_{n}\right\| \rightarrow 0, n \rightarrow \infty
$$

and

$$
\left\|T_{n} w_{n}-x_{n}\right\| \leq\left\|u_{n}-w_{n}\right\|+\left\|w_{n}-x_{n}\right\| \rightarrow 0, n \rightarrow \infty .
$$

From (31), we get

$$
\begin{aligned}
\left\|x_{n+1}-x^{*}\right\|^{2} \leq & (1+\theta)\left\|x_{n}-x^{*}\right\|^{2}-\theta\left\|x_{n-1}-x^{*}\right\|^{2} \\
& +\theta\left[1+\theta-(\theta-1)\left[\frac{(2-\gamma)}{\alpha \gamma}+\frac{(1-\alpha)}{\alpha}\right]\right]\left\|x_{n}-x_{n-1}\right\|^{2}
\end{aligned}
$$

Using Lemma 2.8 in (44) (noting (39)), we get

$$
\lim _{n \rightarrow \infty}\left\|x_{n}-x^{*}\right\|^{2}=l<\infty .
$$

Hence, $\left\{\left\|x_{n}-x^{*}\right\|\right\}$ is bounded. Therefore $\left\{x_{n}\right\}$ is bounded. 
Remark 4.2. It is clear to see that the boundness of $\left\{x_{n}\right\}$ implies that $\left\{w_{n}\right\},\left\{y_{n}\right\}$ and $\left\{T_{n} w_{n}\right\}$ are all bounded.

We are now in position to prove the weak convergence result.

Theorem 4.3. The sequence $\left\{x_{n}\right\}$ generated by Algorithm 3.2 weakly converges to a point in $S$ when Assumptions 3.1 are satisfied.

Proof. By [45], we have that $\lim _{n \rightarrow \infty}\left\|x_{n}-x^{*}\right\|$ exists. Therefore, $\left\{x_{n_{k}}\right\}$ is bounded. Let us prove that any weak cluster point of $\left\{x_{n}\right\}$ is in $S$. Let $\left\{x_{n_{k}}\right\}$ be a subsequence of $\left\{x_{n}\right\}$ that converges weakly to a point $p$ in $H$. Since $\left\{x_{n_{k}}\right\}$ converges weakly to $p$ and $\left\|x_{n}-w_{n}\right\| \rightarrow 0, n \rightarrow \infty$, we obtain that $\left\{w_{n_{k}}\right\}$ converges weakly to $p$. It is immediate that $\left\{w_{n_{k}}\right\}$ converges weakly to $p$. Invoking Assumption 3.1 (c) and (42), we conclude that $p$ belongs to $S$. Then, by Lemma 2.9, we have that $\left\{x_{n}\right\}$ converges weakly to a point in $S$.

Corollary 4.4. Let $\left\{T_{i}\right\}_{i \geq 0} \subset F_{\beta}$, where $\beta \in(0,1)$ and $\cap_{i \geq 0} F\left(T_{i}\right) \neq \emptyset$. Assume that $T_{i}$ is demi-closed for each $i$. Let the sequence $\left\{x_{n}\right\}$ in $H$ be generated by choosing $x_{0}, x_{1} \in H$ and using the recursion

$$
\left\{\begin{array}{l}
w_{n}=x_{n}+\theta\left(x_{n}-x_{n-1}\right) \\
x_{n+1}=(1-\alpha) w_{n}+\alpha \sum_{i=0}^{\infty} \sigma_{i, n} T_{i} w_{n}, \quad n \geq 1,
\end{array}\right.
$$

where $\sigma_{i, n} \in[0, \infty)$ such that $\sum_{i=0}^{\infty} \sigma_{i, n}=1$; for all $i \geq 0,\left\{\sigma_{i, n}\right\}_{n \geq 0}$ is bounded away from zero for $n$ large enough (i.e., $\forall i \geq 0, \exists N_{i} \in \mathbb{N}$ and $\exists \sigma_{i}>0$ such that $\left.\forall n \geq N_{i}, \sigma_{i, n} \geq \sigma_{i}\right) ; \theta \in[0,1)$, and $\alpha \in\left(0, \frac{(1-\theta)^{2}}{\beta \theta(1+\theta)+\beta(1-\theta)^{2}}\right)$. Then $\left\{x_{n}\right\}$ weakly converges to a point in $\cap_{i \geq 0} F\left(T_{i}\right)$.

Proof. Following the same line of arguments as in and of 24, Lemma 4.1, Theorem 4.2], one can show that Assumptions 3.1 hold with $T_{n}=\sum_{i=0}^{\infty} \sigma_{i, n} T_{i}$. The rest of the proof follows from the consequence of Corollary 4.1 and Theorem 4.3 .

Corollary 4.5. Let $\left\{T_{i}\right\}_{i \geq 0} \subset F_{\beta}$, where $\beta \in(0,1)$ and $\cap_{i \geq 0} F\left(T_{i}\right) \neq \emptyset$. Assume that $T_{i}$ is demi-closed for each $i$. Let the sequence $\left\{x_{n}\right\}$ in $H$ be generated by choosing $x_{0}, x_{1} \in H$ and using the recursion

$$
\left\{\begin{array}{l}
w_{n}=x_{n}+\theta\left(x_{n}-x_{n-1}\right) \\
x_{n+1}=(1-\alpha) w_{n}+\alpha\left(\sum_{k=1}^{n} \gamma_{k}\right)^{-1} \sum_{i=1}^{n} \gamma_{i} T_{i} w_{n}, \quad n \geq 1,
\end{array}\right.
$$

where $\gamma_{n} \in(0, \infty)$ with $\sum_{k=1}^{\infty} \gamma_{k}<\infty, \theta \in[0,1)$, and $\alpha \in\left(0, \frac{(1-\theta)^{2}}{\beta \theta(1+\theta)+\beta(1-\theta)^{2}}\right)$. Then $\left\{x_{n}\right\}$ weakly converges to a point in $\cap_{i \geq 0} F\left(T_{i}\right)$.

Proof. Take $\sigma_{i, n}=\frac{\gamma_{i}}{\sum_{k=1}^{n} \gamma_{k}}$ for $1 \leq k \leq n$ and $\sigma_{i, n}=0$ for $i \geq n+1$ in Theorem 4.4. Then $\sum_{i=1}^{\infty} \sigma_{i, n}=1$. Furthermore, since $\sum_{k=1}^{\infty} \gamma_{k}<\infty$, we have for all $i \geq 0$ and large enough $n, \sigma_{i, n} \geq \frac{\gamma_{i}}{\sum_{k=1}^{\infty} \gamma_{k}}>0$. By Theorem 4.4. we have the desired conclusion. 
Corollary 4.6. Let $\left\{T_{i}\right\}_{i \geq 0}$ be an infinite countable family of $\beta$-averaged mappings defined on $H$, where $\beta \in(0,1)$ and $\cap_{i \geq 0} F\left(T_{i}\right) \neq \emptyset$. Let the sequence $\left\{x_{n}\right\}$ in $H$ be generated by choosing $x_{0}, x_{1} \in H$ and using the recursion

$$
\left\{\begin{array}{l}
w_{n}=x_{n}+\theta\left(x_{n}-x_{n-1}\right) \\
x_{n+1}=(1-\alpha) w_{n}+\alpha\left(\sum_{k=1}^{n} \gamma_{k}\right)^{-1} \sum_{i=1}^{n} \gamma_{i} T_{i} w_{n}, \quad n \geq 1,
\end{array}\right.
$$

where $\gamma_{n} \in(0, \infty)$ with $\sum_{k=1}^{\infty} \gamma_{k}<\infty, \theta \in[0,1)$, and $\alpha \in\left(0, \frac{(1-\theta)^{2}}{\beta \theta(1+\theta)+\beta(1-\theta)^{2}}\right)$. Then $\left\{x_{n}\right\}$ weakly converges to a point in $\cap_{i \geq 0} F\left(T_{i}\right)$.

Proof. If $T_{i}$ is $\beta$-averaged on $H$, then $\left\|T_{i} x-T_{i} y\right\|^{2} \leq\|x-y\|^{2}-\frac{1-\beta}{\beta} \| x-y-\left(T_{i} x-\right.$ $\left.T_{i} y\right) \|^{2}, \forall x, y \in H$. Consequently,

$$
\left\|T_{i} x-x^{*}\right\|^{2} \leq\left\|x-x^{*}\right\|^{2}-\frac{1-\beta}{\beta}\left\|x-T_{i} x\right\|^{2}, \forall x \in H, x^{*} \in \cap_{i \geq 0} F\left(T_{i}\right) .
$$

Thus, we get that $T_{i} \in F_{\beta}$. Since $T_{i}$ is nonexpansive for each $i \geq 0$, we have that each $T_{i}$ is demi-closed. Hence, by invoking Corollary 4.5, we obtain the desired conclusion.

\section{Remark 4.7.}

(i) All the results in this Section still hold if we replace the constant $\theta \in[0,1)$ in Algorithm 3.2 with $\theta_{n} \in[0,1)$ such that $0 \leq \theta_{n} \leq \theta_{n+1}<1$. We only stick to constant $\theta \in[0,1]$ for the sake of brevity.

(ii) Our result also complement the recent results given in [2, 4, 5, 10, 24, 28] and many other related recent results in the literature.

\section{Some Applications}

\subsection{Three-Operator Splitting Method}

We study this inclusion problem:

$$
\text { Find } x \in H \text { such that } 0 \in A x+B x+C x \text {, }
$$

where $A, B$ and $C$ are maximal monotone operators defined on $H$ and in addition, $C$ is $\eta$-inverse strongly monotone (i.e., $\eta$-cocoercive). Suppose the set of solutions to problem (47) is denoted by $\operatorname{zer}(A+B+C)$. Define

$$
T:=J_{\gamma A} \circ\left(2 J_{\gamma B}-I-\gamma C \circ J_{\gamma B}\right)+I-J_{\gamma B}, \gamma>0 .
$$

Then we obtain the following result.

Proposition 5.1. [16, Proposition 2.1] Assume that $A$ and $B$ are maximal monotone operators and $C$ a $\eta$-cocoercive mapping. Take $\gamma \in(0,2 \eta)$. Then $T$ in (48) is a $\beta$-averaged mapping with $\beta:=\frac{2 \eta}{4 \eta-\gamma}<1$ and $\operatorname{zer}(A+B+C)=J_{\gamma B}(F(S))$. Furthermore, if $\gamma \in(0,2 \eta \epsilon)$, where $\epsilon \in(0,1)$, then for all $w, z \in H$,

$$
\begin{aligned}
\|T z-T w\|^{2} \leq & \|z-w\|^{2}-\frac{1-\beta}{\beta}\|(I-T) z-(I-T) w\|^{2} \\
& -\gamma\left(2 \eta-\frac{\gamma}{\epsilon}\right)\left\|C \circ J_{\gamma B}(z)-T \circ J_{\gamma B}(w)\right\|^{2} .
\end{aligned}
$$


The focus here is to adapt Algorithm 3.2 to solve (47). So, set $w_{n}=x_{n}+\theta\left(x_{n}-\right.$ $\left.x_{n-1}\right), y_{n}=J_{\gamma B}\left(w_{n}\right)$ and $z_{n}=J_{\gamma A}\left(2 y_{n}-w_{n}-\gamma C y_{n}\right)$. Then from Algorithm 3.2, with $T=J_{\gamma A} \circ\left(2 J_{\gamma B}-I-\gamma C \circ J_{\gamma B}\right)+I-J_{\gamma B}$, we get $x_{n+1}=w_{n}-\alpha\left(z_{n}-y_{n}\right)$. Thus, we obtain the following method to solve (47): take $x_{0}, x_{1} \in H$ and generate $\left\{x_{n}\right\}$ by

$$
\left\{\begin{array}{l}
w_{n}=x_{n}+\theta\left(x_{n}-x_{n-1}\right) \\
y_{n}=J_{\gamma B}\left(w_{n}\right) \\
z_{n}=J_{\gamma A}\left(2 y_{n}-w_{n}-\gamma C y_{n}\right) \\
x_{n+1}=w_{n}-\alpha\left(z_{n}-y_{n}\right),
\end{array}\right.
$$

where $\theta \in[0,1)$, and $\alpha \in\left(0, \frac{(1-\theta)^{2}(4 \eta-\gamma)}{2 \eta \theta(1+\theta)+2 \eta(1-\theta)^{2}}\right)$. Consequently, we have the following weak convergence result for inclusion problem (47).

Theorem 5.2. Assume $\operatorname{zer}(A+B+C) \neq \emptyset$. Let $\gamma \in(0,2 \eta \epsilon)$, where $\epsilon \in(0,1)$, $\theta \in[0,1)$, and $\alpha \in\left(0, \frac{(1-\theta)^{2}(4 \eta-\gamma)}{2 \eta \theta(1+\theta)+2 \eta(1-\theta)^{2}}\right)$. Choose $x_{0}, x_{1} \in H$, and let $\left\{x_{n}\right\}$ be the sequence generated by (50). Then the following hold:

(i) $\left\{x_{n}\right\}$ weakly converges to a fixed point $z^{*}$ of $S$ and $J_{\gamma B}\left(z^{*}\right)$ solves inclusion problem (47);

(ii) $y_{n}-z_{n} \rightarrow 0, n \rightarrow \infty$;

(iii) $\left\{C y_{n}\right\}$ converges strongly to $C x^{*}$ for any $x^{*} \in \operatorname{zer}(A+B+C)$;

(iv) the sequences $\left\{y_{n}\right\}$ and $\left\{z_{n}\right\}$ weakly converge to $J_{\gamma B}\left(z^{*}\right) \in \operatorname{zer}(A+B+C)$;

$(v)$ both $\left\{y_{n}\right\}$ and $\left\{z_{n}\right\}$ converge strongly to a point in $\operatorname{zer}(A+B+C)$ if any of the following is satisfied:

(a) $A$ is uniformly monotone on every nonempty bounded subset of $\operatorname{dom}(A)(A$ is uniformly monotone if there exists $\phi: \mathbb{R}_{+} \rightarrow[0, \infty]$ such that $\phi(0)=0$ and for all $u \in A x, v \in A y,\langle x-y, u-v\rangle \geq \phi(\|x-y\|)$.);

(b) $B$ is uniformly monotone on every nonempty bounded subset of $\operatorname{dom}(B)$;

(c) $C$ is demiregular at every point $y \in \operatorname{zer}(A+B+C)(C$ is demiregular at $x \in \operatorname{dom}(C)$ if for all $u \in C x$ and for all $\left(x_{n}, u_{n}\right) \in \operatorname{gra}(C)$ with $x_{n}-x$, and $u_{n} \rightarrow u$, we have $x_{n} \rightarrow x$.

Proof. Using Theorem 4.3 and [16, Lemma 2.2], we obtain the desired conclusion of (i).

(ii) From (42) in Lemma 4.1 we get $\left\|y_{n}-z_{n}\right\|=\left\|w_{n}-T w_{n}\right\| \rightarrow 0, n \rightarrow \infty$.

(iii) Observe that

$$
\begin{aligned}
\left\|x_{n+1}-z^{*}\right\|^{2}= & (1-\alpha)\left\|w_{n}-z^{*}\right\|^{2}+\alpha\left\|T w_{n}-z^{*}\right\|^{2} \\
& -\alpha(1-\alpha)\left\|w_{n}-T w_{n}\right\|^{2} .
\end{aligned}
$$

Using (49) in (51),

$$
\left\|x_{n+1}-z^{*}\right\|^{2} \leq(1-\alpha)\left\|w_{n}-z^{*}\right\|^{2}+\alpha\left\|w_{n}-z^{*}\right\|^{2}
$$




$$
\begin{aligned}
& -\frac{\alpha(1-\beta)}{\beta}\left\|w_{n}-T w_{n}\right\|^{2}-\gamma \alpha\left(2 \eta-\frac{\gamma}{\epsilon}\right)\left\|C y_{n}-C J_{\gamma B}\left(z^{*}\right)\right\|^{2} \\
& -\alpha(1-\alpha)\left\|w_{n}-T w_{n}\right\|^{2} .
\end{aligned}
$$

This implies that

$$
\begin{aligned}
& \gamma \alpha\left(2 \eta-\frac{\gamma}{\epsilon}\right)\left\|C y_{n}-C J_{\gamma B}\left(z^{*}\right)\right\|^{2} \leq\left\|w_{n}-z^{*}\right\|^{2} \\
& -\left\|x_{n+1}-z^{*}\right\|^{2} \\
= & (1+\theta)\left\|x_{n}-z^{*}\right\|^{2}-\theta\left\|x_{n-1}-z^{*}\right\|^{2} \\
& +\theta(1+\theta)\left\|x_{n}-x_{n-1}\right\|^{2}-\left\|x_{n+1}-z^{*}\right\|^{2} \\
\leq & \left\|x_{n}-z^{*}\right\|^{2}-\left\|x_{n+1}-z^{*}\right\|^{2}+\theta\left[\left\|x_{n}-z^{*}\right\|^{2}-\left\|x_{n-1}-z^{*}\right\|^{2}\right]_{+} \\
& +\theta(1+\theta)\left\|x_{n}-x_{n-1}\right\|^{2} .
\end{aligned}
$$

Therefore,

$$
\begin{aligned}
& \sum_{i=n}^{\infty}\left\|C y_{i}-C J_{\gamma B}\left(z^{*}\right)\right\|^{2} \leq \frac{1}{\gamma \lambda_{n}\left(2 \eta-\frac{\gamma}{\epsilon}\right)}\left[\left\|x_{n}-z^{*}\right\|^{2}\right. \\
& +\sum_{i=n}^{\infty}\left[\left\|x_{i}-z^{*}\right\|^{2}-\left\|x_{i-1}-z^{*}\right\|^{2}\right]_{+} \\
& \left.+2 \sum_{i=n}^{\infty}\left\|x_{i}-x_{i-1}\right\|^{2}\right] .
\end{aligned}
$$

Using Lemma 2.8 in (44), we have $\sum_{i=n}^{\infty}\left[\left\|x_{i}-z^{*}\right\|^{2}-\left\|x_{i-1}-z^{*}\right\|^{2}\right]_{+}<\infty$. Also by (39), we get $\sum_{i=n}^{\infty}\left\|x_{i}-x_{i-1}\right\|^{2}<\infty$. Therefore, we obtain $\lim _{n \rightarrow \infty}\left\|C y_{n}-C J_{\gamma B}\left(z^{*}\right)\right\|=$ 0 . This completes (iii).

(iv) Note that

$$
\begin{aligned}
\left\|y_{n}-J_{\gamma B}\left(z^{*}\right)\right\| & =\left\|J_{\gamma B}\left(w_{n}\right)-J_{\gamma B}\left(z^{*}\right)\right\| \\
& \leq\left\|w_{n}-z^{*}\right\| \leq\left\|(1+\theta) x_{n}-\theta x_{n-1}-z^{*}\right\| .
\end{aligned}
$$

Therefore, $\left\{y_{n}\right\}$ is bounded since $\left\{x_{n}\right\}$ is bounded by Corollary 4.1. Now, suppose $z$ is the sequential weak cluster point of $\left\{y_{n}\right\}$. Then there exists $\left\{y_{n_{k}}\right\} \subset\left\{y_{n}\right\}$ such that $y_{n_{k}} \rightarrow z, k \rightarrow \infty$. Pick $x^{*} \in \operatorname{zer}(A+B+C)$. Since $C$ is maximal monotone, $C y_{n} \rightarrow C x^{*}$ and $y_{n_{k}} \rightarrow z$, it follows from the weak-to-strong sequential closedness of $C$ that $C z=C x^{*}$ by [6. Proposition 20.33 (ii)] and so $C y_{n_{k}} \rightarrow C z$.

Denote $u_{n}:=\frac{1}{\gamma}\left(w_{n}-y_{n}\right) \in B y_{n}$, and $v_{n}:=\frac{1}{\gamma}\left(2 y_{n}-w_{n}-\gamma C y_{n}-z_{n}\right) \in A z_{n}$.

Then we get $y_{n_{k}} \rightarrow z, z_{n_{k}} \rightarrow z, C y_{n_{k}} \rightarrow C z, w_{n_{k}} \rightarrow z^{*}, u_{n_{k}} \rightarrow \frac{1}{\gamma}\left(z^{*}-z\right)$ and $v_{n_{k}} \rightarrow \frac{1}{\gamma}\left(z-z^{*}-\gamma C z\right)$. Applying [6, Proposition 25.5] to $\left(z_{n_{k}}, v_{n_{k}}\right) \in \operatorname{gra} A$, $\left(y_{n_{k}}, u_{n_{k}}\right) \in B$ and $\left(y_{n_{k}}, C y_{n_{k}}\right) \in C$ shows that $z \in \operatorname{zer}(A+B+C), z^{*}-z \in \gamma B z$ and $z-z^{*}-\gamma C z \in \gamma A z$. Hence, we have shown that $z=J_{\gamma B}\left(z^{*}\right)$ and so $z$ is unique weak cluster point of $\left\{y_{n}\right\}$. Therefore, $\left\{y_{n}\right\}$ weakly converges to $J_{\gamma B}\left(z^{*}\right)$ by 
[6, Lemma 2.38]. Furthermore, $\left\{z_{n}\right\}$ weakly converges to $J_{\gamma B}\left(z^{*}\right)$ since $\left\{y_{n}\right\}$ weakly converges to $J_{\gamma B}\left(z^{*}\right)$ and $y_{n}-z_{n} \rightarrow 0, n \rightarrow \infty$.

(vi) Let $x^{*}=J_{\gamma B}\left(z^{*}\right), u^{*}=\frac{1}{\gamma}\left(z^{*}-x^{*}\right) \in B x^{*}$ and $v^{*}=\frac{1}{\gamma}\left(x^{*}-z^{*}\right)-C x^{*} \in A x^{*}$.

(a) Since $B+C$ is monotone and $\left(y_{n}, u_{n}\right) \in B$, we get

$$
\left\langle y_{n}-x^{*}, u_{n}+C y_{n}-\left(u^{*}+C x^{*}\right)\right\rangle \geq 0, \forall n \geq 1 .
$$

Consider the set $S:=\left\{x^{*}\right\} \cup\left\{z_{n}\right\}$. Then there exists $\Phi_{A}: \mathbb{R}_{+} \rightarrow[0, \infty]$ that vanishes only at 0 such that

$$
\begin{aligned}
\gamma \Phi_{A}\left(\left\|z_{n}-x^{*}\right\|\right) \leq & \gamma\left\langle z_{n}-x^{*}, v_{n}-x^{*}\right\rangle \\
& +\gamma\left\langle y_{n}-x^{*}, u_{n}+C y_{n}-\left(u^{*}+C x^{*}\right)\right\rangle \\
= & \gamma\left\langle z_{n}-y_{n}, v_{n}-v^{*}\right\rangle+\gamma\left\langle z_{n}-x^{*}, v_{n}-v^{*}\right\rangle \\
& +\gamma\left\langle y_{n}-x^{*}, u_{n}+C y_{n}-\left(u^{*}+C x^{*}\right)\right\rangle \\
= & \gamma\left\langle z_{n}-y_{n}, v_{n}-v^{*}\right\rangle+\gamma\left\langle y_{n}-x^{*}, v_{n}+u_{n}+C y_{n}\right\rangle \\
= & \left\langle y_{n}-z_{n}, y_{n}-\gamma v_{n}-\left(x^{*}-\gamma v^{*}\right)\right\rangle \\
= & \left\langle y_{n}-z_{n}, w_{n}-z^{*}\right\rangle+\gamma\left\langle y_{n}-z_{n}, C y_{n}-C x^{*}\right\rangle \\
& +\left\langle y_{n}-z_{n}, w_{n}-z^{*}\right\rangle \\
\leq & \left\langle y_{n}-z_{n}, w_{n}-z^{*}\right\rangle+\gamma\left\langle y_{n}-z_{n}, C y_{n}-C x^{*}\right\rangle \rightarrow 0, n \rightarrow \infty,
\end{aligned}
$$

since $y_{n}-z_{n}=w_{n}-T w_{n} \rightarrow 0, n \rightarrow \infty, w_{n} \rightarrow z^{*}$ and $C y_{n} \rightarrow C x^{*}, n \rightarrow \infty$. Also, $y_{n} \rightarrow x^{*}$ since $z_{n}-y_{n} \rightarrow 0, n \rightarrow \infty$.

(b) Since $A$ is monotone, we get $\left\langle z_{n}-x^{*}, v_{n}-x^{*}\right\rangle \geq 0, n \geq 1$. Observe also that $B+C$ is uniformly monotone on all bounded sets. Let us consider the bounded set $S:=\left\{x^{*}\right\} \cup\left\{y_{n}\right\}$. Then there exists an increasing function $\Phi_{B}: \mathbb{R}_{+} \rightarrow[0, \infty]$ that vanishes only at 0 such that

$$
\begin{aligned}
\Phi_{B}\left(\left\|y_{n}-x^{*}\right\|\right) \leq & \gamma\left\langle z_{n}-x^{*}, v_{n}-x^{*}\right\rangle \\
& +\gamma\left\langle y_{n}-x^{*}, u_{n}+C y_{n}-\left(u^{*}+C x^{*}\right)\right\rangle \rightarrow 0, n \rightarrow \infty,
\end{aligned}
$$

by part (a) above. Hence we get $y_{n} \rightarrow x^{*}, n \rightarrow \infty$.

(c) Observe that $C y_{n} \rightarrow C x^{*}$ and $y_{n} \rightarrow x^{*}$. Then by demiregularity of $C$, we get $y_{n} \rightarrow x^{*}, n \rightarrow \infty$.

\subsection{Application to Optimization Problems}

Consider the composite convex optimization problem:

$$
\min _{x \in H} F(x)=f(x)+g(x),
$$

where $f, g$ are proper, lower-semicontinuous convex functions taking values in $(-\infty, \infty]$ with $f$ being $L$-smooth (i.e., the gradient $\nabla f$ of $f$ is $L$-Lipschitz continuous). The proximal-gradient algorithm

$$
x_{n+1}=\operatorname{prox}_{\lambda_{n} g}\left(x_{n}-\lambda_{n} \nabla f\left(x_{n}\right)\right),
$$

where $\lambda \in\left(0, \frac{2}{L}\right)$. 


\section{Remark 5.3.}

Observe that $\nabla f$ is $L$-Lipschitz continuous implies that $\nabla f$ is $\frac{1}{L}$-ism [?]. This further implies that $\lambda \nabla f$ is $\frac{1}{\lambda L}$-ism. So by [31, Proposition 3.4(iii)], $I-\lambda \nabla f$ is $\frac{\lambda L}{2}$-averaged. Now since Prox $\operatorname{Pr}_{\lambda g}$ is $\frac{1}{2}$-averaged, we see from [31, Proposition 3.2(iv)] that $T:=\operatorname{prox}_{\lambda g}(I-\lambda \nabla f)$ is $\frac{2+\lambda L}{4}$ - averaged.

Using Remark 5.3 and Algorithm 3.2 , we have the following relaxed inertial proximalgradient method to solve problem (52):

$$
\left\{\begin{array}{l}
w_{n}=x_{n}+\theta\left(x_{n}-x_{n-1}\right) \\
x_{n+1}=(1-\alpha) w_{n}+\alpha \operatorname{prox}_{\lambda_{n} g}\left(w_{n}-\lambda_{n} \nabla f\left(w_{n}\right)\right),
\end{array}\right.
$$

where $\theta \in[0,1), 0<a \leq \liminf _{n \rightarrow \infty} \lambda_{n} \leq \limsup _{n \rightarrow \infty} \lambda_{n} \leq b<\frac{2}{L}$ and $\alpha \in$ $\left(0, \frac{4(1-\theta)^{2}}{(2+b L)\left(\theta(1+\theta)+(1-\theta)^{2}\right)}\right)$. Consequently, we have the following weak convergence result for inclusion problem (47).

Theorem 5.4. Consider the composite optimization problem (52), where $f, g$ are proper, lower-semicontinuous convex functions with $f L$-smooth. Suppose the set of solutions of (52), denoted by $S$, is nonempty. Suppose $\theta \in[0,1), 0<a \leq$ $\liminf _{n \rightarrow \infty} \lambda_{n} \leq \lim \sup _{n \rightarrow \infty} \lambda_{n} \leq b<\frac{2}{L}$ and $\alpha \in\left(0, \frac{4(1-\theta)^{2}}{(2+b L)\left(\theta(1+\theta)+(1-\theta)^{2}\right)}\right)$. Then the sequence $\left\{x_{n}\right\}$ generated by Algorithm 54 converges weakly to a point in $S$.

Proof. Define

$$
T=\operatorname{prox}_{\lambda g}(I-\lambda \nabla f) .
$$

Then $T$ is $\frac{2+\lambda L}{4}$ - averaged mapping and hence averaged quasi-nonexpansive mapping. Furthermore, $S=F(T)$ and Algorithm 3.2 reduces to Algorithm 54. Then by Theorem 4.3 we have that $\left\{x_{n}\right\}$ converges to a point in $S$.

The give the following remark regarding our method (50) and some related methods.

\section{Remark 5.5.}

(a) Suppose $\alpha=1, \theta=0$ and $g$ is an indicator function in Algorithm 54 , then Algorithm 54 reduces to [31, (2),(3)]. Also, if we take $\theta=0$ and $g$ is an indicator function in Algorithm 54, then Algorithm 54 reduces to [31, (18)].

(b) If we take $\theta=0$ in Algorithm 54, then Algorithm 54 reduces to [31, (18)].

Now, let study the composite nonconvex optimization problem:

$$
\min _{x \in H} F(x)=f(x)+g(x),
$$

where $f, g$ are proper, lower-semicontinuous functions taking values in $(-\infty, \infty]$ with $f L$-smooth and $g$ being continuous when restricted to its domain. We propose the following relaxed inertial nonconvex proximal gradient algorithm:

$$
\left\{\begin{array}{l}
w_{n}=x_{n}+\theta\left(x_{n}-x_{n-1}\right) \\
x_{n+1} \in(1-\alpha) w_{n}+\alpha \operatorname{prox}_{\lambda g}\left(w_{n}-\lambda \nabla f\left(w_{n}\right)\right)
\end{array}\right.
$$


where $\theta \in[0,1), \lambda \in\left(0, \frac{1}{L}\right)$ and $\alpha \in\left(0, \frac{4(1-\theta)^{2}}{(2+\lambda L)\left(\theta(1+\theta)+(1-\theta)^{2}\right)}\right)$. Using similar arguments in [3], 26, Theorem 2.4] and Theorem 4.3, we have the following weak convergence result for composite nonconvex optimization problem (55).

Theorem 5.6. Suppose $f, g$ are proper, lower-semicontinuous functions taking values in $(-\infty, \infty]$ with $f L$-smooth and $g$ being continuous when restricted to its domain. Assume that $F=f+g$ and $F$ is a KL function and bounded from below. Choose $\theta \in[0,1), \lambda \in\left(0, \frac{1}{L}\right)$ and $\alpha \in\left(0, \frac{4(1-\theta)^{2}}{(2+\lambda L)\left(\theta(1+\theta)+(1-\theta)^{2}\right)}\right)$. Then $\left\{x_{n}\right\}$ generated by (56) converges weakly to a critical point of $F$.

Let us apply our results to reweighted $l_{Q}$-regularization in recovering a signal from the noised linear system

$$
A x=b+e,
$$

where $A \in \mathbb{R}^{m \times k}$ is the measurement (or sensing) matrix, $b \in \mathbb{R}^{m}$ is the observation and $e$ stands for the errors (noise). Let us look at the regularized optimization problem

$$
\min _{x \in \mathbb{R}^{k}} F_{\wedge, Q}(x):=\frac{1}{2}\|A x-b\|_{2}^{2}+\sum_{j=1}^{k} \gamma_{j}\left|x_{j}\right|^{q_{j}},
$$

where $\wedge:=\left(\gamma_{1}, \ldots, \gamma_{k}\right)$ and $Q:=\left(q_{1}, \ldots, q_{k}\right)$ are the parameter sets of weights and exponents, respectively. We treat the sparseness more effectively by allocating different weights to magnitudes of different components of the signal raised to different powers. We now adapt our methods in Algorithm 54 and Algorithm 56 to solve problem (58).

For the convex case [30] with $1 \leq q_{j} \leq 2, j=1, \ldots, k$, we have the following adapted method using the ideas in [26] by writing $F(x):=F_{\wedge, Q}(x), f(x):=\frac{1}{2}\|A x-b\|_{2}^{2}$ and $g(x):=\sum_{j=1}^{k} \gamma_{j}\left|x_{j}\right|^{q_{j}}$ with $\nabla f(x)=A^{T}(A x-b)$ and $L=\|A\|_{2}^{2}$.

$$
\left\{\begin{array}{l}
w_{n}=x_{n}+\theta\left(x_{n}-x_{n-1}\right) \\
x_{n+1}=(1-\alpha) w_{n}+\alpha \operatorname{prox}_{\lambda g}\left(w_{n}-\lambda A^{T}\left(A w_{n}-b\right)\right),
\end{array}\right.
$$

where $\theta \in[0,1), \lambda \in\left(0, \frac{2}{\|A\|_{2}^{2}}\right)$ and $\alpha \in\left(0, \frac{4(1-\theta)^{2}}{\left(2+\lambda\|A\|_{2}^{2}\right)\left(\theta(1+\theta)+(1-\theta)^{2}\right)}\right)$.

In this case, the proximal mapping $\operatorname{prox}_{\lambda g}$ is evaluated by this result.

Lemma 5.7. (see [26, Lemma 3.4]) Assume $q_{j} \in[1,2]$ and $\lambda>0$. Then for $x=\left(x_{1}, x_{2}, \ldots, x_{k}\right)^{T} \in \mathbb{R}^{k}$, we have

$$
\operatorname{prox}_{\lambda g}(x)=\left(\operatorname{prox}_{\lambda \gamma_{1} \mid . q^{q_{1}}}\left(x_{1}\right), \ldots, \operatorname{prox}_{\lambda \gamma_{k}||^{q_{k}}}\left(x_{k}\right)\right)^{T},
$$

where

$$
\operatorname{prox}_{\lambda \gamma_{j}|.| q_{j}}\left(x_{j}\right)=\left\{\begin{array}{l}
\operatorname{shrink}_{\lambda \gamma_{j}}\left(x_{j}\right), \quad q_{j}=1 \\
\phi_{j}^{-1}\left(x_{j}\right), \quad 1<q_{j}<2 \\
\frac{1}{2 \lambda \gamma_{j}+1} x_{j}, \quad q_{j}=2,
\end{array}\right.
$$


with

$$
\operatorname{shrink}_{\lambda \gamma_{j}}\left(x_{j}\right)=\operatorname{sgn}\left(x_{j}\right) \max \left\{\left|x_{j}\right|-\lambda \gamma_{j}, 0\right\}, j=1,2, \ldots, k
$$

being the soft-shrinkage operator and

$$
\phi_{j}:=\lambda \gamma_{j} q_{j} \operatorname{sgn}(t)|t|^{q_{j}-1}+t \quad t \in \mathbb{R}, j=1,2, \ldots, k .
$$

Then by Algorithm 59 we have the following result.

Theorem 5.8. Let $q_{j} \in[1,2], j=1,2, \ldots, k$ and $\lambda>0$. The sequence of iterates $\left\{x_{n}\right\}$ generated by Algorithm (59) converges to a solution of (58).

Now for the nonconvex approach to solving problem (58) with $q_{j} \in(0,1), j=$ $1,2, \ldots, k$. The following lemma is important for this case.

Lemma 5.9. (see [26, Lemma 3.6]) Suppose $g$ is defined by $g(x):=\sum_{j=1}^{k} \gamma_{j}\left|x_{j}\right|^{q_{j}}$, where $\gamma_{j}>0$ and $q_{j} \in(0,1), j=1,2, \ldots, k$. Then $\operatorname{prox}_{\lambda g}(x)$ is nonempty for every $x \in \mathbb{R}^{k}$ and given by the formula

$$
\operatorname{prox}_{\lambda g}(x)=\left(\operatorname{prox}_{\lambda g_{1}}\left(x_{1}\right), \ldots, \operatorname{prox}_{\lambda g_{k}}\left(x_{k}\right)\right)^{T},
$$

where, for $j=1,2, \ldots, k, g_{j}\left(x_{j}\right)=\gamma_{j}\left|x_{j}\right|^{q_{j}}$ and

$$
\operatorname{prox}_{\lambda g_{j}}\left(x_{j}\right)= \begin{cases}0, & \left|x_{j}\right| \leq \tau_{j} \\ \phi_{j}^{-1}\left(x_{j}\right), & \left|x_{j}\right|>\tau_{j}\end{cases}
$$

where the threshold $\tau_{j}$ is given by

$$
\tau_{j}:=\frac{2-q_{j}}{2-2 q_{j}}\left(2 \lambda \gamma_{j}\left(1-q_{j}\right)\right)^{\frac{1}{2-q_{j}}}
$$

and the function $\phi_{j}$ is given by (60) with $q_{j} \in(0,1), j=1,2, \ldots, k$.

We have the following result for the $l_{Q}$-regularization $(58)$ in the full nonconvex case.

Theorem 5.10. Let us consider the $l_{Q}$-regularization (58) in the full nonconvex case with $q_{j} \in(0,1), j=1,2, \ldots, k$. Take $x_{0}, x_{1} \in \mathbb{R}^{k}$ and suppose $\left\{x_{n}\right\}$ be generated by the inertial nonconvex $P G A$ :

$$
\left\{\begin{array}{l}
w_{n}=x_{n}+\theta\left(x_{n}-x_{n-1}\right) \\
x_{n+1} \in(1-\alpha) w_{n}+\alpha \operatorname{prox}_{\lambda g}\left(w_{n}-\lambda A^{T}\left(A w_{n}-b\right)\right),
\end{array}\right.
$$

where $\theta \in[0,1), \lambda \in\left(0, \frac{1}{\|A\|_{2}^{2}}\right)$ and $\alpha \in\left(0, \frac{4(1-\theta)^{2}}{\left(2+\lambda\|A\|_{2}^{2}\right)\left(\theta(1+\theta)+(1-\theta)^{2}\right)}\right)$.

Then $\left\{x_{n}\right\}$ converges to a critical point of (58) with finite length in the sense that $\sum_{n=1}^{\infty}\left\|x_{n}-x_{n-1}\right\|^{2}<\infty$. 
We perform some numerical examples to show performances of relaxed inertial proximal-gradient method (54) and relaxed inertial nonconvex proximal gradient algorithm (56). First, we consider the convex case, that is, each component of the exponent set $\mathrm{Q}$ is bigger than or equal to one and give performance of (54). Finally, we consider the nonconvex case where all components of $Q$ are strictly between zero and one, and we demonstrate the convergence performance of (56).

In all our examples, we compare relaxed inertial proximal-gradient method (54) with [26. (3.15)], [26, (3.24)] We choose different $q_{j}=1, q_{j}=\frac{3}{2}, q_{j}=2, \forall j=1,2, \ldots, k$ and $\gamma=\frac{1}{\|A\|_{2}^{2}}$ in different dimensions of $A \in \mathbb{R}^{200 \times 800}, A \in \mathbb{R}^{300 \times 1000}, A \in \mathbb{R}^{400 \times 1200}$ and $A \in \mathbb{R}^{800 \times 2600}$ problem $(58)$.

Let a sparse signal $x_{e}$ be generated randomly with sparsity of $1.2 \%$ and set $b=A x_{e}$.

We discuss the relative error and least squared error, and select the appropriate number of iteration steps respectively. We take $\lambda=0.9 \frac{2}{\|A\|_{2}^{2}}, \theta=0.9$ and $\alpha=$ $0.9 \frac{4(1-\theta)^{2}}{\left(2+\lambda\|A\|_{2}^{2}\right)\left(\theta(1+\theta)+(1-\theta)^{2}\right)}$ for $(54)$ in this case, and take $\mu=0.9 \frac{2}{\|A\|_{2}^{2}}$ for [26, (3.15)]. But when comparing the least squared error in $q_{j}=\frac{3}{2}$ and $q_{j}=2, \forall j=1,2, \ldots, k$, we choose $\lambda=0.6 \frac{2}{\|A\|_{2}^{2}}, \theta=0.6$ and $\alpha=0.9 \frac{4(1-\theta)^{2}}{\left(2+\lambda\|A\|_{2}^{2}\right)\left(\theta(1+\theta)+(1-\theta)^{2}\right)}$ for (54).

Now we make comparision of two algorithms with the initial values $x_{0}=x_{1}=y_{0}=$ $0.5 * \operatorname{rand}(k, 1)$. Let $m=300$ and $k=1000$. In Fig. 1, we plot the signal to recover. In Fig. 2, the left one is the recovered signal of Algorithm (54) when $q_{j}=1$, and the right one is the recovered signal of Algorithm [26, (3.15)]. In Fig. 3, the left one is relative error $\left(\frac{\left\|x_{n}-x_{e}\right\|_{2}}{\left\|x_{n}\right\|_{2}}\right)$, the other is least squared error $\left(\left\|A x_{n}-b\right\|_{2}\right)$. In Fig. 4 , it is the recovered signals of Algorithm (54) and Algorithm [26, (3.15)] when $q_{j}=\frac{3}{2}$. In Fig. 5, it is the relative error and least squared error. In Fig. 66, it is the recovered signals of Algorithm (54) and Algorithm [26, (3.15)] when $q_{j}=2$. In Fig. 77, it is the relative error and least squared error.

In Table 1, we use the stopping criterion $\frac{\left\|x_{n}-x_{e}\right\|_{2}}{\left\|x_{n}\right\|_{2}}<10^{-3}$ when $q_{j}=1$. In Table 2 , we use the stopping criterion $\left\|A x_{n}-b\right\|_{2}<0.3$ when $q_{j}=1$.

We also compare relaxed inertial nonconvex proximal gradient algorithm (56) with [26, (3.23)], [26, (3.26)]. Here we consider $q_{j}=\frac{1}{2}, q_{j}=\frac{2}{3}, \forall j=1,2, \ldots, k$ and $\gamma=\frac{1}{2\|A\|_{2}^{2}}$ in different dimensions of $A \in \mathbb{R}^{200 \times 800}, A \in \mathbb{R}^{300 \times 1000}, A \in \mathbb{R}^{400 \times 1200}$ and $A \in \mathbb{R}^{800 \times 2600}$ problem (58).

We take $\lambda=0.9 \frac{2}{\|A\|_{2}^{2}}, \theta=0.9$ and $\alpha=0.9 \frac{4(1-\theta)^{2}}{\left(2+\lambda\|A\|_{2}^{2}\right)\left(\theta(1+\theta)+(1-\theta)^{2}\right)}$ for 556 in this case, and take $\mu=0.9 \frac{2}{\|A\|_{2}^{2}}$ for [26, (3.23)]. We discuss the relative error and least squared error, and select the appropriate number of iteration steps respectively.

We now make comparision of two algorithms with the initial values $x_{0}=x_{1}=y_{0}=$ 


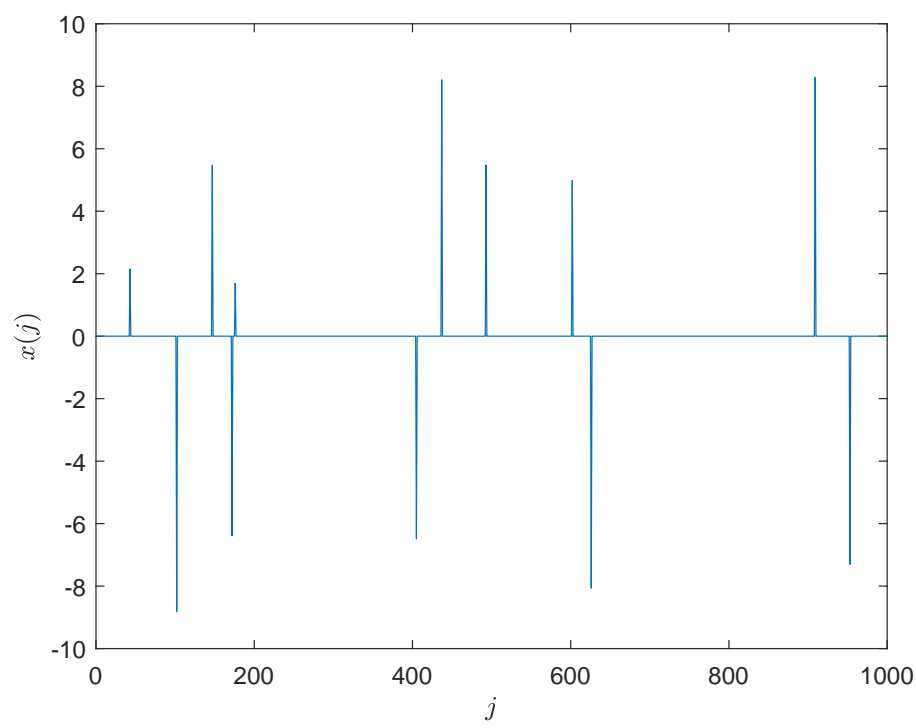

Figure 1: Sparse model $x$.
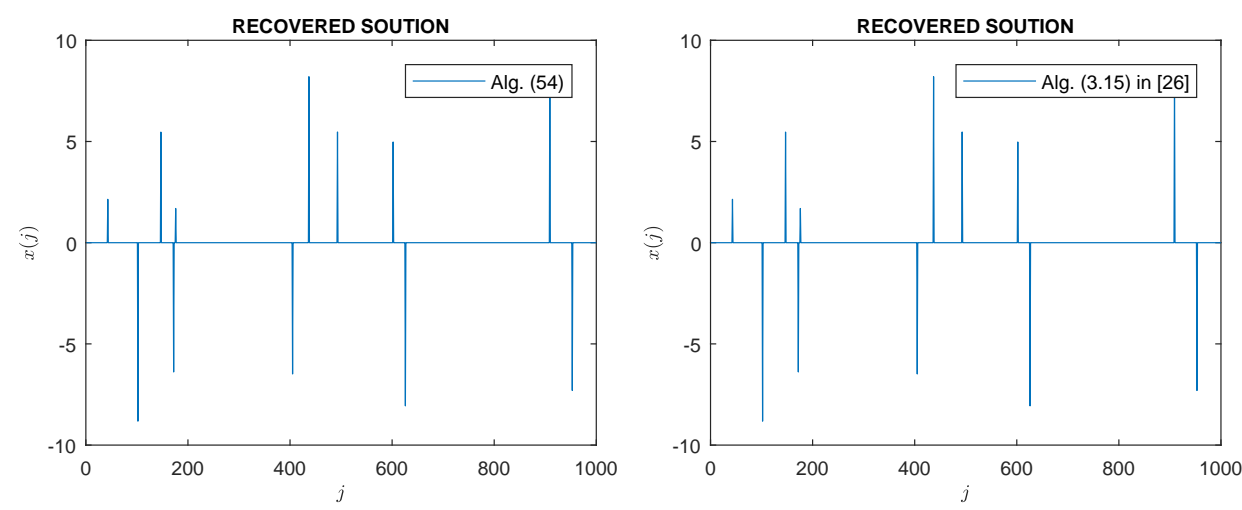

Figure 2: Recovered signals of Algorithm (54) and Algorithm [26, (3.15)] $\left(q_{j}=1\right)$.

$0.5 * \operatorname{rand}(k, 1)$. Let $m=300$ and $k=1000$. In Fig. 8 , the left one is the recovered signal of Algorithm (56) when $q_{j}=\frac{1}{2}$, and the right one is the recovered signal of Algorithm [26, (3.23)]. In Fig. 9, the left one is relative error, and the other is least squared error. In Fig. 10, it is the recovered signals of Algorithm (56) and Algorithm [26, (3.23)] when $q_{j}=\frac{2}{3}$. In Fig. 11, it is the relative error and least squared error.

The "." in tables means that the number of iterations is more than $10^{4}$. In Table 3. we use the stopping criterion $\frac{\left\|x_{n}-x_{e}\right\|_{2}}{\left\|x_{n}\right\|_{2}}<10^{-3}$ when $q_{j}=\frac{1}{2}$ and $q_{j}=\frac{2}{3}$. In Table 4. we use the stopping criterion $\left\|A x_{n}-b\right\|_{2}<0.2$ when $q_{j}=\frac{1}{2}$ and $q_{j}=\frac{2}{3}$. As shown in Fig. 9. Fig. 11, Table 3 and Table 4 , Algorithm (56) behaves better than Algorithm [26, (3.23)].

Remark 6.1. We have the following observations from the figures and tables above. 

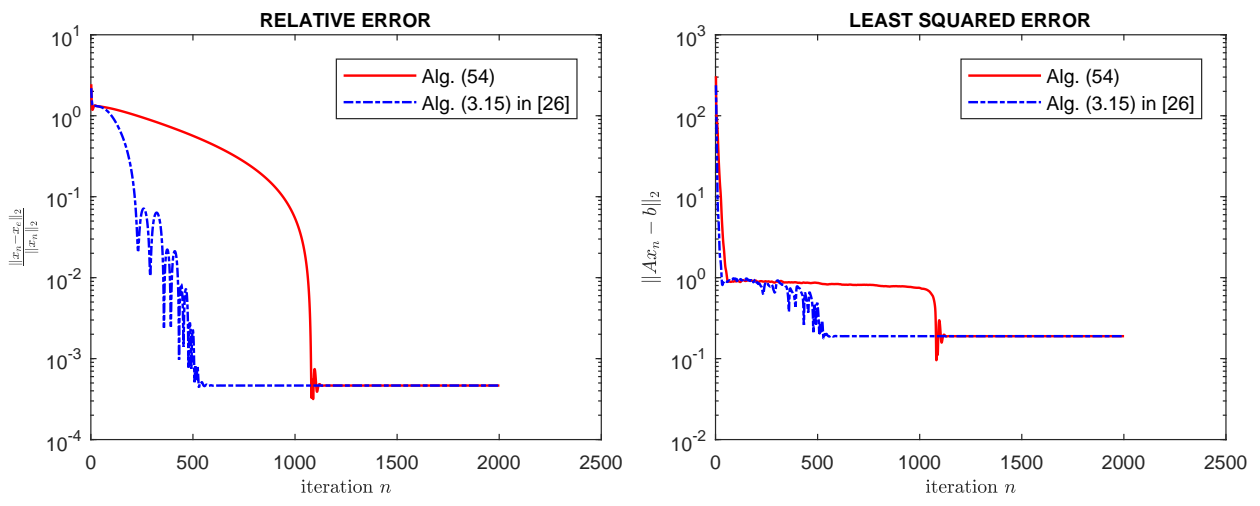

Figure 3: Relative error and least squared error with Algorithm (54) and Algorithm [26, (3.15)] $\left(q_{j}=1\right)$.

Table 1: Comparison of Algorithm (54) and Algorithm [26, (3.15)] when $q_{j}=1$ and $\frac{\left\|x_{n}-x_{e}\right\|_{2}}{\left\|x_{n}\right\|_{2}}<10^{-3}$.

\begin{tabular}{rccccc}
\hline \multirow{2}{*}{ Problem size } & $m$ & 200 & 300 & 400 & 800 \\
\cline { 2 - 6 } & $k$ & 800 & 1000 & 1200 & 2600 \\
\hline \hline \multirow{2}{*}{ Alg. (54) } & Iter & 832 & 1145 & 1315 & 2781 \\
\cline { 2 - 6 } & CPU time & 1.3420 & 3.1228 & 5.7473 & 45.0203 \\
\hline Alg. (3.15) in [26] & Iter & 398 & 460 & 501 & 741 \\
\cline { 2 - 6 } & CPU time & 0.6514 & 1.2623 & 2.2978 & 11.4652 \\
\hline
\end{tabular}

Table 2: Comparison of Algorithm (54) and Algorithm [26, (3.15)] when $q_{j}=1$ and $\left\|A x_{n}-b\right\|_{2}<0.3$.

\begin{tabular}{cccccc}
\hline \multirow{2}{*}{ Problem size } & $m$ & 200 & 300 & 400 & 800 \\
\cline { 2 - 6 } & $k$ & 800 & 1000 & 1200 & 2600 \\
\hline \hline \multirow{2}{*}{ Alg. (54) } & Iter & 1062 & 1142 & 1132 & 2294 \\
\cline { 2 - 6 } & CPU time & 1.4644 & 2.8824 & 4.6961 & 38.0790 \\
\hline \multirow{2}{*}{ Alg. (3.15) in [26] } & Iter & 414 & 444 & 450 & 775 \\
\cline { 2 - 6 } & CPU time & 0.6531 & 1.1796 & 1.8435 & 12.7082 \\
\hline \hline
\end{tabular}



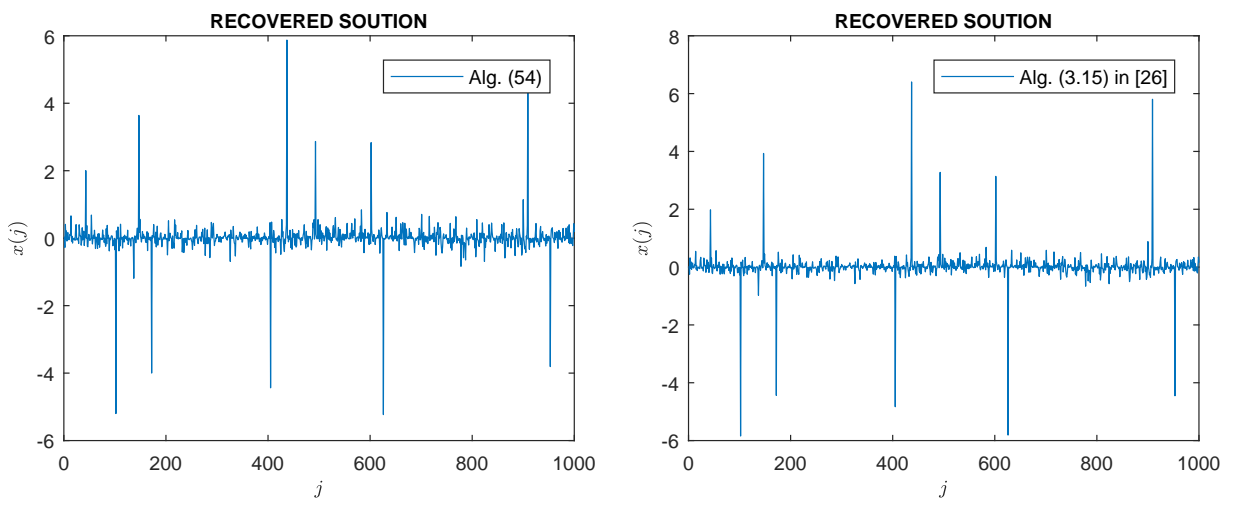

Figure 4: Recovered signals of Algorithm (54) and Algorithm [26, (3.15)] $\left(q_{j}=\frac{3}{2}\right)$.
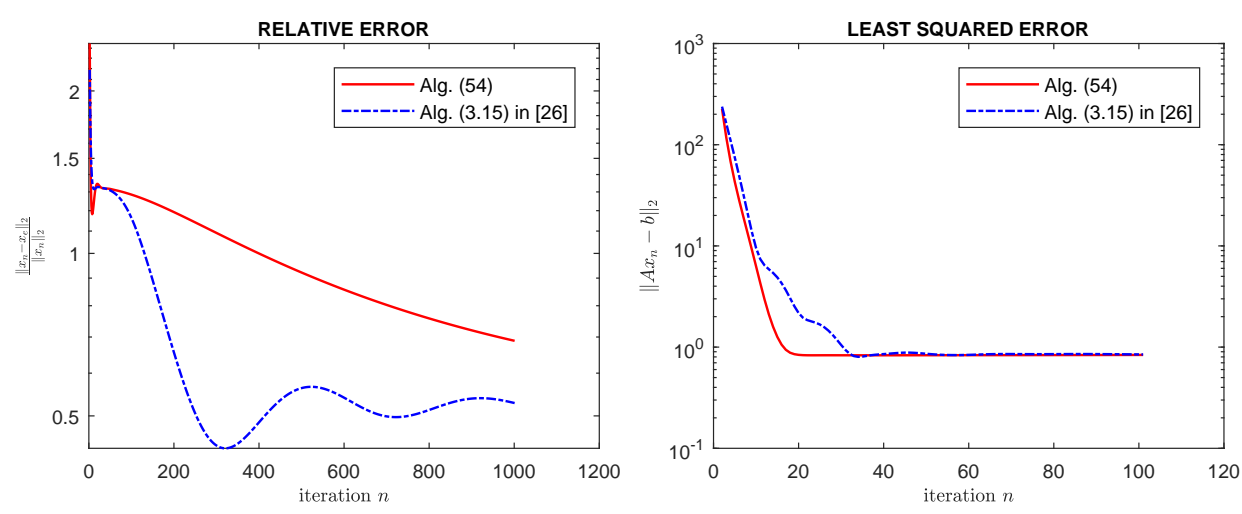

Figure 5: Relative error and least squared error with Algorithm (54) and Algorithm [26, (3.15)] $\left(q_{j}=\frac{3}{2}\right)$.

1. From Figure 3, Table 1 and Table 2, one can see that [26, (3.15)] outperforms our Algorithm (54) in terms of number of iterations and CPU time when $q_{j}=1$.

2. From Figure 9, Figure 11, Table 3 and Table 4, we see that Algorithm (56) outperforms [26, (3.23)] in terms of number of iterations and CPU time when $q_{j}=\frac{1}{2}$ and $q_{j}=\frac{2}{3}$.

\section{$7 \quad$ Final Remarks}

This paper introduces an over-relaxed version of inertial fixed point method for approximation of common fixed point of countable family of averaged quasi-nonexpansive mappings in real Hilbert spaces. Several proposed methods in the literature are recovered as special cases of our new proposed method. Weak convergence result is obtained using the new method and some applications to three-operator splitting 

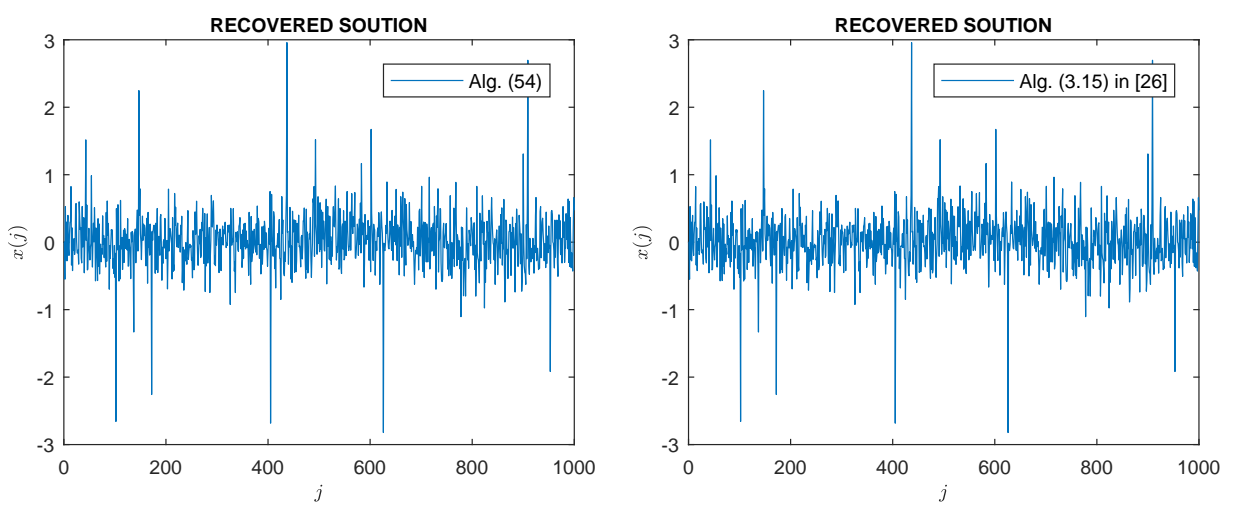

Figure 6: Recovered signals of Algorithm (54) and Algorithm [26, (3.15)] $\left(q_{j}=2\right)$.
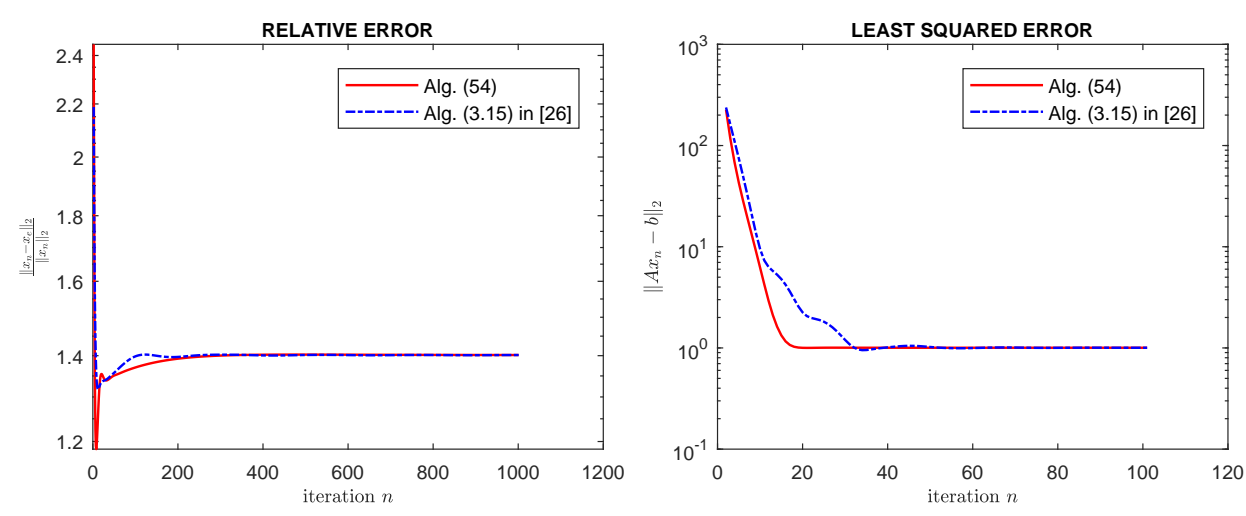

Figure 7: Relative error and least squared error with Algorithm (54) and Algorithm [26, (3.15)] $\left(q_{j}=2\right)$.

method and composite convex and non-convex optimization problems are given. Finally, numerical implementations from sparse signal recovery are given to illustrate the effectiveness of our method. We have not studied the convergence rate results of the proposed method and certain complexity results including priori and posteriori error estimates are not given. These are interesting projects we shall consider in the future.

\section{Compliance with ethical standards}

Ethical approval All the authors approved the final version of this manuscript.

Funding details No funding was received for this manuscript.

Conflict of interest The authors declare that they have no conflict of interest. 

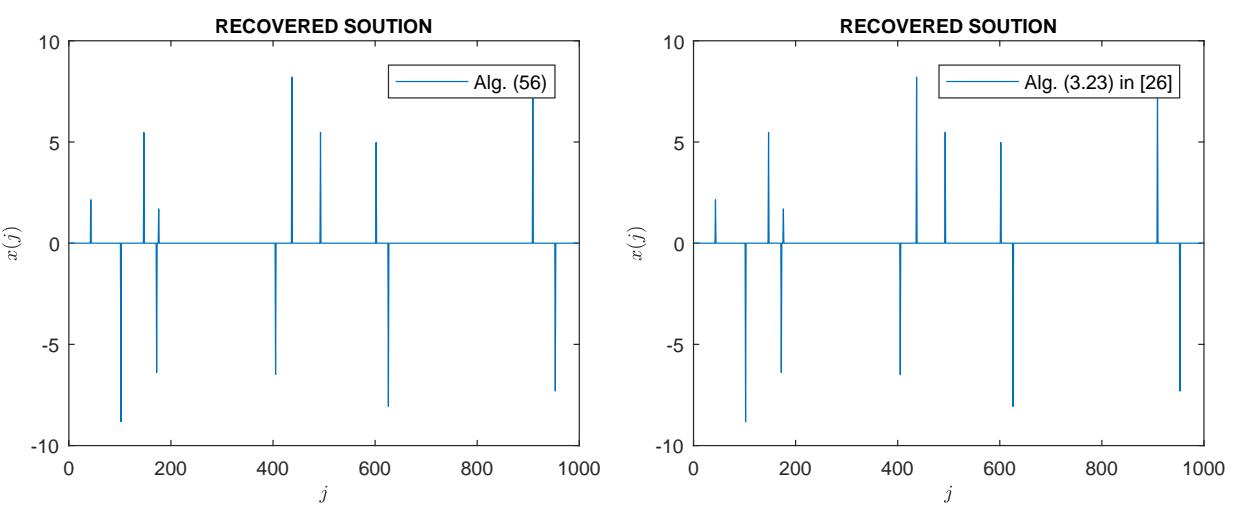

Figure 8: Recovered signals of Algorithm (56) and Algorithm [26, (3.23)] $\left(q_{j}=\frac{1}{2}\right)$.
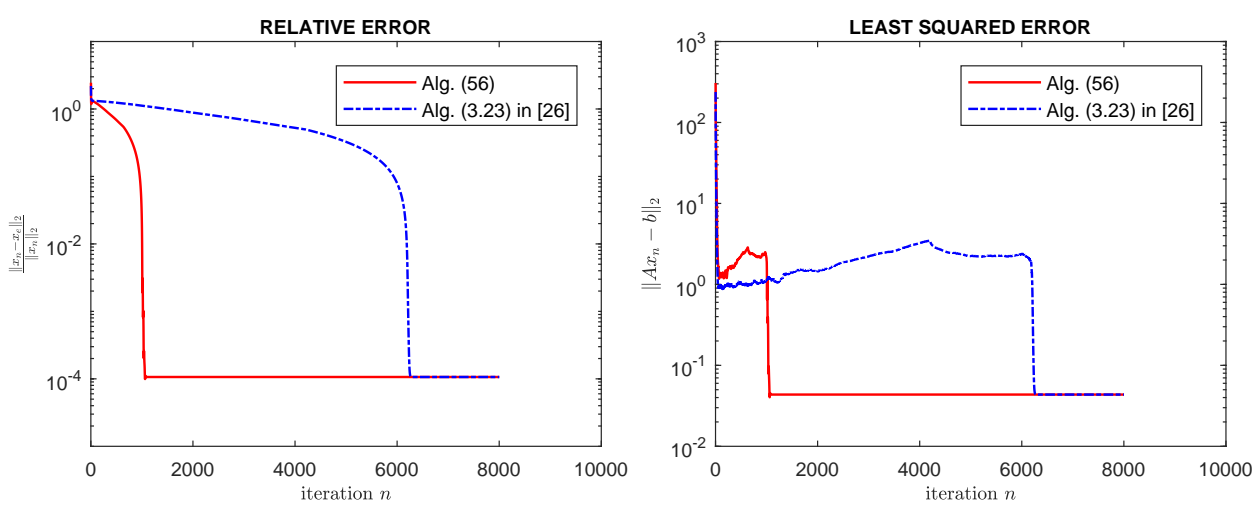

Figure 9: Relative error and least squared error with Algorithm (56) and Algorithm [26, (3.23)] $\left(q_{j}=\frac{1}{2}\right)$.

Informed Consent All the authors understand the purpose of the research and their individual role.

Author's contribution All the authors contributed equally to the final version of the manuscript.

\section{References}

[1] F. Alvarez; Weak convergence of a relaxed and inertial hybrid projectionproximal point algorithm for maximal monotone operators in Hilbert space, SIAM J. Optim. 14, (2003) 773-782. 8

[2] F. Alvarez, and H. Attouch; An inertial proximal method for maximal monotone operators via discretization of a nonlinear oscillator with damping, Set-Valued 

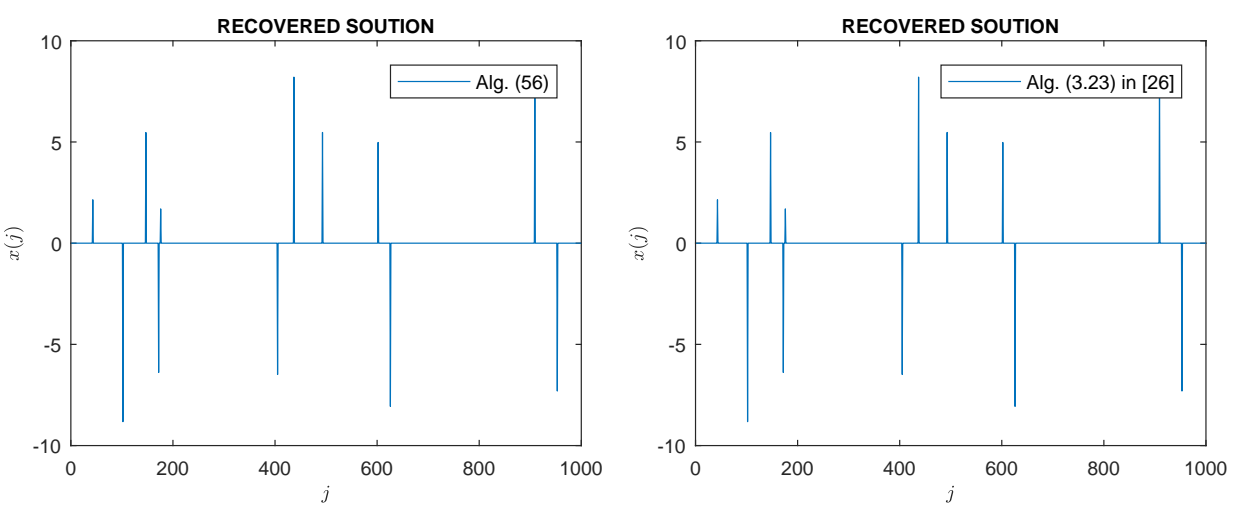

Figure 10: Recovered signals of Algorithm (56) and Algorithm [26, $(3.23)]\left(q_{j}=\frac{2}{3}\right)$.
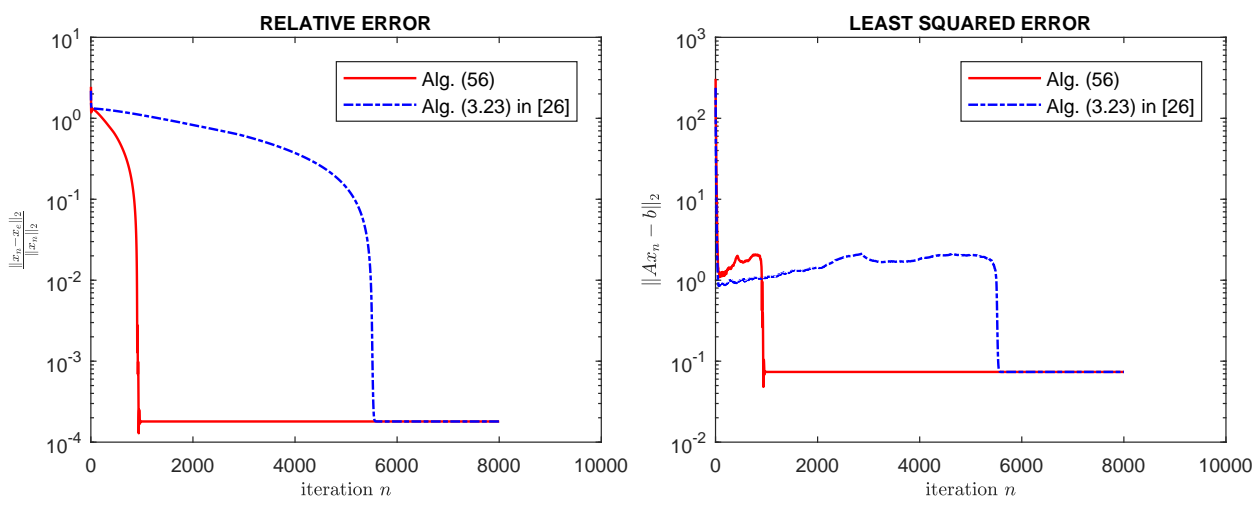

Figure 11: Relative error and least squared error with Algorithm (56) and Algorithm [26, (3.23)] $\left(q_{j}=\frac{2}{3}\right)$.

Var. Anal. 9 (2001), 3-11. 2, 13

[3] H. Attouch, J. Bolte, and B. F. Svaiter; Convergence of descent methods for semi-algebraic and tame problems: proximal algorithms, forward-backward splitting, and regularized Gauss-Seidel methods, Math. Program. 137 (2013), no. 1-2, Ser. A, 91-129. 18

[4] H. Attouch, X. Goudon, and P. Redont; The heavy ball with friction. I. The continuous dynamical system, Commun. Contemp. Math. 2 (1) (2000), 1-34. 2. 13

[5] H. Attouch, and M.O. Czarnecki; Asymptotic control and stabilization of nonlinear oscillators with non-isolated equilibria, J. Differential Equations 179 (1) (2002), 278-310. 2, 13 
Table 3: Comparison of Algorithm (56) and Algorithm [26, (3.23)] when $\frac{\left\|x_{n}-x_{e}\right\|_{2}}{\left\|x_{n}\right\|_{2}}<10^{-3}$.

\begin{tabular}{|c|c|c|c|c|c|c|}
\hline \multicolumn{2}{|c|}{ Problem size } & \multirow[t]{2}{*}{ Algorithm } & \multicolumn{2}{|c|}{$q_{j}=\frac{1}{2}$} & \multicolumn{2}{|c|}{$q_{j}=\frac{2}{3}$} \\
\hline$m$ & $k$ & & Iter & CPU time & Iter & CPU time \\
\hline \multirow{2}{*}{200} & \multirow{2}{*}{800} & Alg. (56) & 925 & 1.4350 & 884 & 1.5720 \\
\hline & & Alg. (3.23) in [26] & 5474 & 8.5014 & 5271 & 9.8548 \\
\hline \multirow{2}{*}{300} & \multirow{2}{*}{1000} & Alg. (56) & 876 & 2.3703 & 828 & 2.5854 \\
\hline & & Alg. (3.23) in [26] & 5214 & 15.1491 & 4919 & 16.0460 \\
\hline \multirow{2}{*}{400} & \multirow{2}{*}{1200} & Alg. (56) & 914 & 3.8244 & 872 & 4.0959 \\
\hline & & Alg. (3.23) in [26] & 5515 & 24.7928 & 5230 & 27.9722 \\
\hline \multirow{2}{*}{800} & \multirow{2}{*}{2600} & Alg. (56) & 2267 & 39.5340 & 2133 & 36.6619 \\
\hline & & Alg. (3.23) in [26] & - & - & - & - \\
\hline
\end{tabular}

Table 4: Comparison of Algorithm (56) and Algorithm [26, (3.23)] when $\left\|A x_{n}-b\right\|_{2}<0.2$.

\begin{tabular}{|c|c|c|c|c|c|c|}
\hline \multicolumn{2}{|c|}{ Problem size } & \multirow[t]{2}{*}{ Algorithm } & \multicolumn{2}{|c|}{$q_{j}=\frac{1}{2}$} & \multicolumn{2}{|c|}{$q_{j}=\frac{2}{3}$} \\
\hline$m$ & $k$ & & Iter & CPU time & Iter & CPU time \\
\hline \multirow{2}{*}{200} & \multirow{2}{*}{800} & Alg. (56) & 695 & 1.0945 & 650 & 1.2479 \\
\hline & & Alg. (3.23) in [26] & 4069 & 6.5346 & 3791 & 7.2952 \\
\hline \multirow{2}{*}{300} & \multirow{2}{*}{1000} & Alg. (56) & 641 & 1.7988 & 579 & 1.8828 \\
\hline & & Alg. (3.23) in [26] & 3806 & 10.8794 & 3417 & 11.1920 \\
\hline \multirow{2}{*}{400} & \multirow{2}{*}{1200} & Alg. (56) & 639 & 2.7879 & 632 & 3.1308 \\
\hline & & Alg. (3.23) in [26] & 3687 & 17.5580 & 3653 & 19.1432 \\
\hline \multirow{2}{*}{800} & \multirow{2}{*}{2600} & Alg. (56) & 2004 & 34.7211 & 1907 & 39.0407 \\
\hline & & Alg. (3.23) in [26] & - & - & - & - \\
\hline
\end{tabular}

[6] H. H. Bauschke, and P. L. Combettes; Convex Analysis and Monotone Operator Theory in Hilbert Spaces, CMS Books in Mathematics, Springer, New York (2011). 2, 3, 4, 15, 16 
[7] A. Beck, and M. Teboulle; A fast iterative shrinkage-thresholding algorithm for linear inverse problems, SIAM J. Imaging Sci. 2 (1) (2009), 183-202. 2

[8] V. Berinde; Iterative Approximation of Fixed Points, Lecture Notes in Mathematics 1912, Springer, Berlin, 2007. 2

[9] R. I. Boţ, E. R. Csetnek, and C. Hendrich; Inertial Douglas-Rachford splitting for monotone inclusion problems, Appl. Math. Comput. 256 (2015), 472-487. 2

[10] J. M. Borwein, G. Li, and M. K. Tam; Convergence rate analysis for averaged fixed point iterations in common fixed point problems, SIAM J. Optim. 27 (2017), 1-33. 2, 8, 13

[11] X. Cai, G. Gu and B. He; On the $O(1 / t)$ convergence rate of the projection and contraction methods for variational inequalities with Lipschitz continuous monotone operators, Comput. Optim. Appl. 57 (2014), 339-363. 4

[12] A. Cegielski; Iterative Methods for Fixed Point Problems in Hilbert Spaces; Lecture Notes in Mathematics 2057, Springer, Berlin, 2012. 2

[13] S. S. Chang, Y. J. Cho, and H. Zhou (eds.); Iterative Methods for Nonlinear Operator Equations in Banach Spaces, Nova Science, Huntington, NY, 2002. 2

[14] C.-S. Chuang, and W. Takahashi; Weak convergence theorems for families of nonlinear mappings with generalized parameters, Numer. Funct. Anal. Optim. 36 (2015), 41-54. 8

[15] E. Corman, and X. Yuan; A generalized proximal point algorithm and its convergence rate estimate, SIAM J. Optim. 24 (2014), 1614-1638. 2, 8

[16] D. Damek, and Y. Watao; A three-operator splitting scheme and its optimization applications, Set-Valued Var. Anal. 25 (2017), 829-858. 13, 14

[17] Y. Dong; Comments on "the proximal point algorithm revisited", J. Optim. Theory Appl. 116 (2015), 343-349. 2, 8

[18] B. S. He; A class of projection and contraction methods for monotone variational inequalities, Appl. Math. Optim. 35 (1997), 69-76. 4

[19] B. S. He, and X. M. Yuan; On the convergence rate of Douglas-Rachford operator splitting method, Math. Program. 153 (2015), 715-722. 2, 8

[20] F. Jules and P. E. Maingé; Numerical approaches to a stationary solution of a second order dissipative dynamical system, Optimization 51 (2002), 235-255. 2

[21] P.-E. Maingé; Convergence theorems for inertial KM-type algorithms, J. Comput. Appl. Math. 219 (2008), 223-236. 2, 7

[22] P. E. Maingé; Regularized and inertial algorithms for common fixed points of nonlinear operators, J. Math. Anal. Appl. 344 (2008), 876-887. 2 
[23] P. E. Maingé; Convergence theorems for inertial KM-type algorithms, J. Comput. Appl. Math. 219 no. 1, (2008), 223-236. 2, 7

[24] P. E. Maingé; Inertial iterative process for fixed points of certain quasinonexpansive mappings, Set-Valued Var. Anal. 15 (2007), 67-79. 2, 8, 12, 13

[25] Z. Opial; Weak convergence of the sequence of successive approximations for nonexpansive mappings, Bull. Am. Math. Soc. 73 (1967), 591-597. 7

[26] B. Peng, and H.-K. Xu; Proximal methods for reweighted $l_{Q}$-regularization of sparse signal recovery, Appl. Math. Comput. 386 (2020), 125408, 15 pp. 18, 19. 20, 21, 22, 23, 24, 25, 26, 27,

[27] B. T. Polyak; Some methods of speeding up the convergence of iterarive methods, Zh. Vychisl. Mat. Mat. Fiz. 4 (1964), 1-17. 2

[28] Y. Shehu; Convergence Rate Analysis of Inertial Krasnoselskii-Mann-type Iteration with Applications, Numer. Funct. Anal. Optim. 39 (2018), 1077-1091. 13

[29] B. F. Svaiter; On weak convergence of the Douglas-Rachford method, SIAM J. Control Optim. 49 (2011), 280-287. 2

[30] S. Voronin, and I. Daubechies; An iteratively reweighted least squares algorithm for sparse regularization, Functional analysis, harmonic analysis, and image processing: a collection of papers in honor of Bjórn Jawerth, 391-411, Contemp. Math., 693, Amer. Math. Soc., Providence, RI, 2017. 18

[31] H.-K. Xu; Averaged mappings and the gradient-projection algorithm, J. Optim. Theory Appl. 150 (2011), 360-378. 8, 17 
Figures

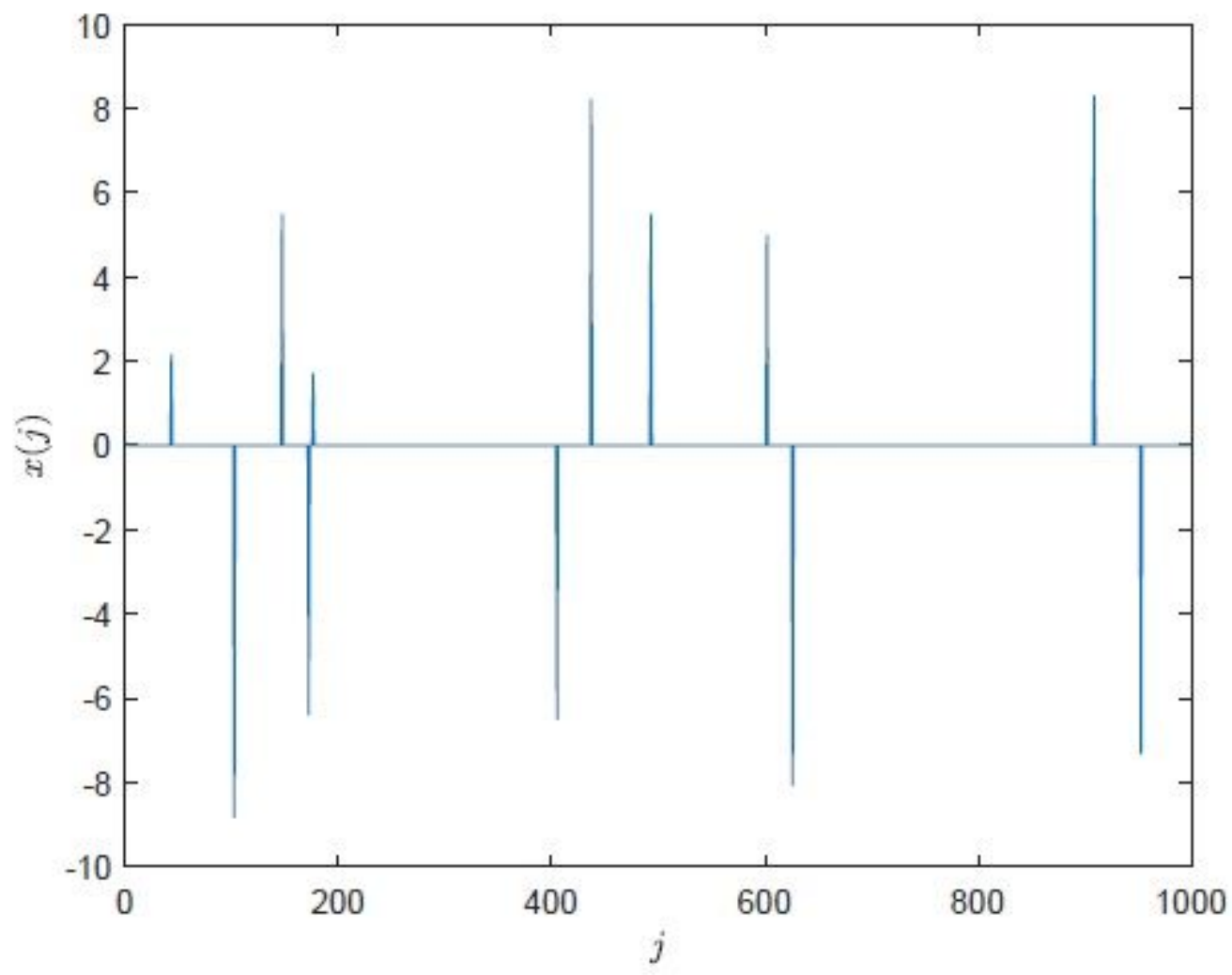

Figure 1

Sparse model $\mathrm{x}$.
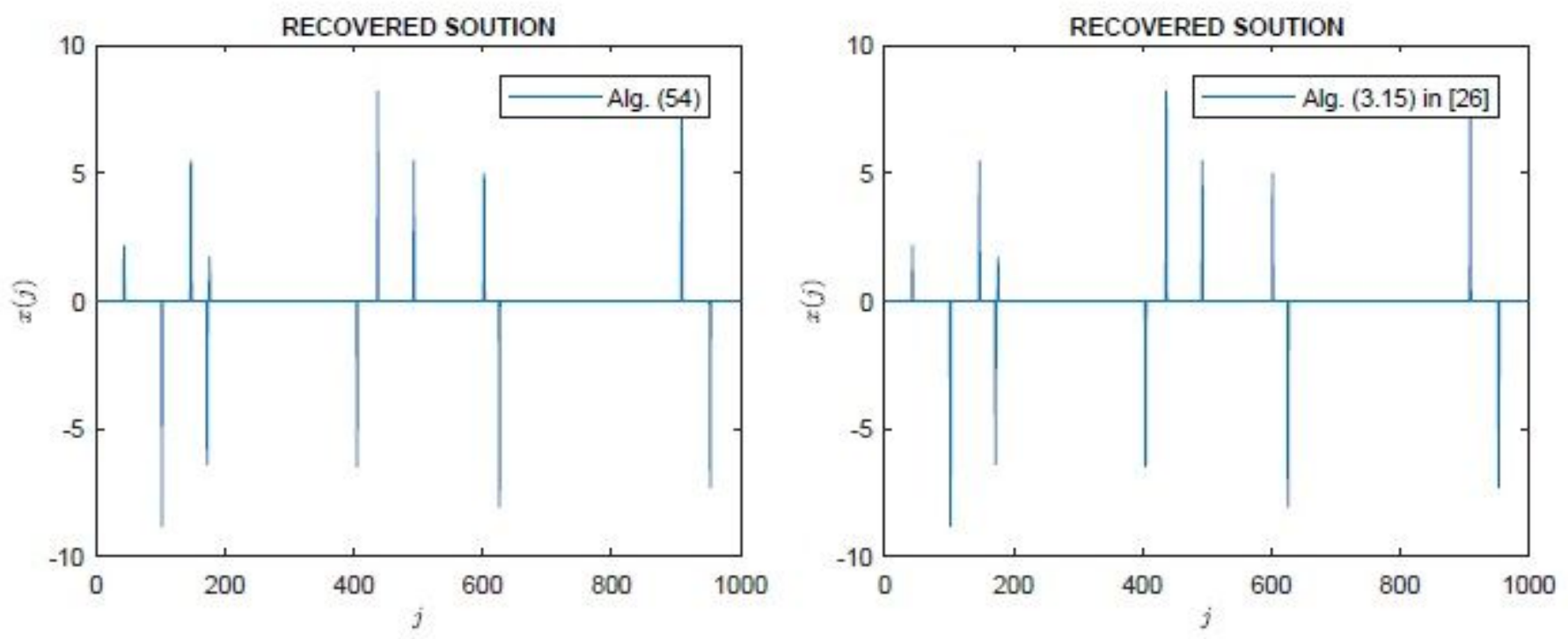

Figure 2

Recovered signals of Algorithm (54) and Algorithm [26, (3.15)] (qj = 1). 

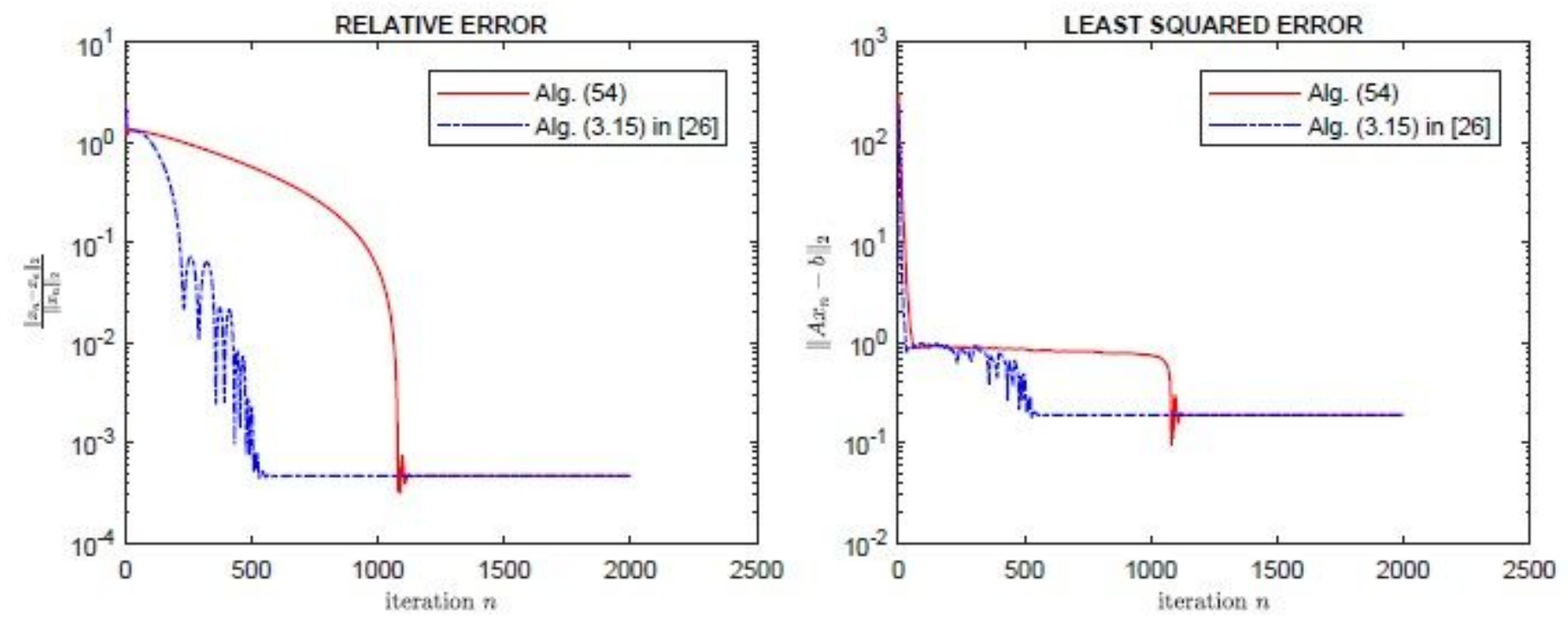

Figure 3

Relative error and least squared error with Algorithm (54) and Algorithm [26, (3.15)] (qj = 1).
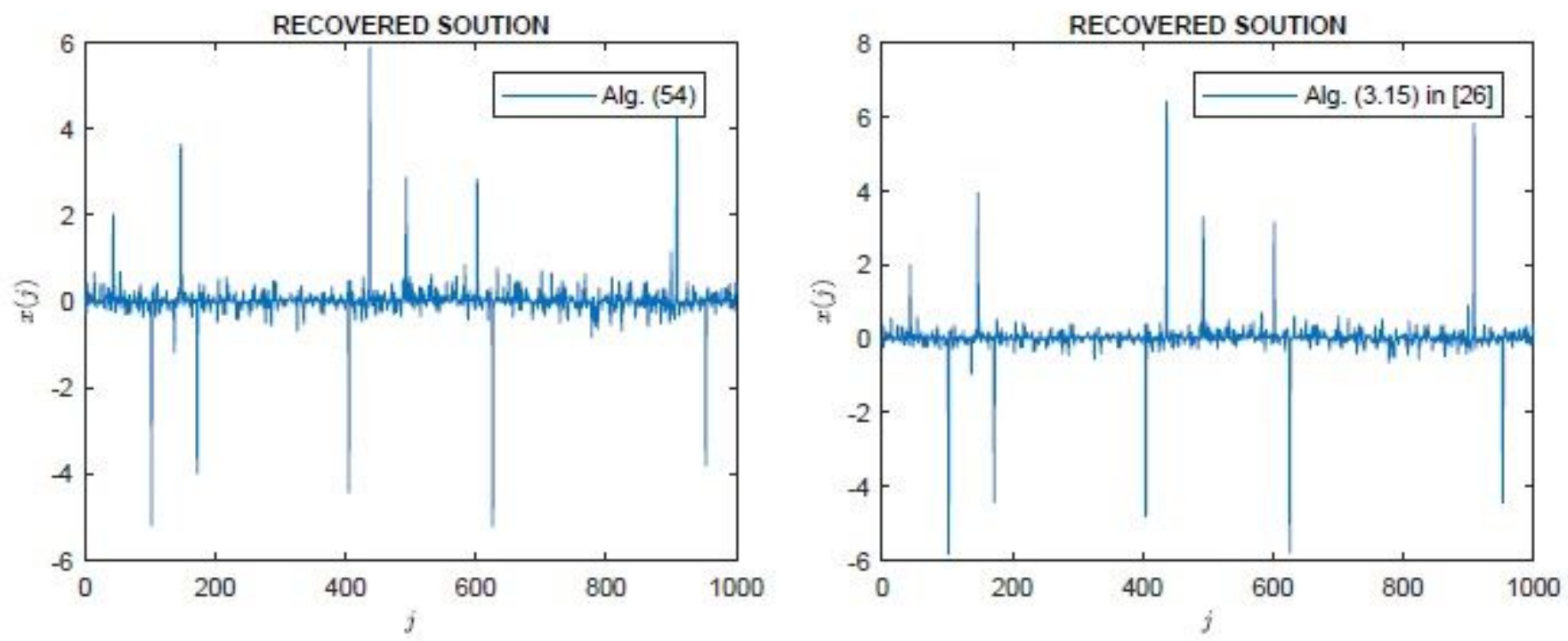

Figure 4

Recovered signals of Algorithm (54) and Algorithm [26, (3.15)] (qj = 3/2 ). 

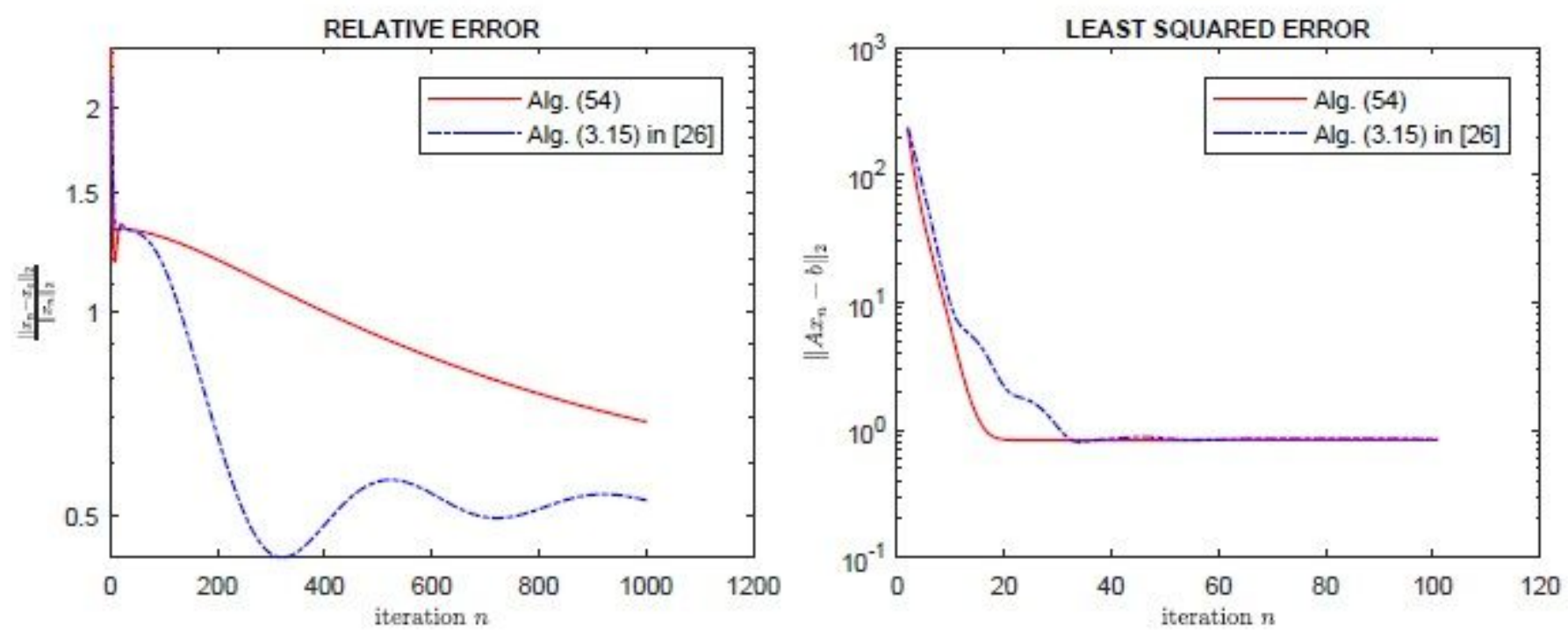

\section{Figure 5}

Relative error and least squared error with Algorithm (54) and Algorithm [26, (3.15)] (qj = 3/2 ).
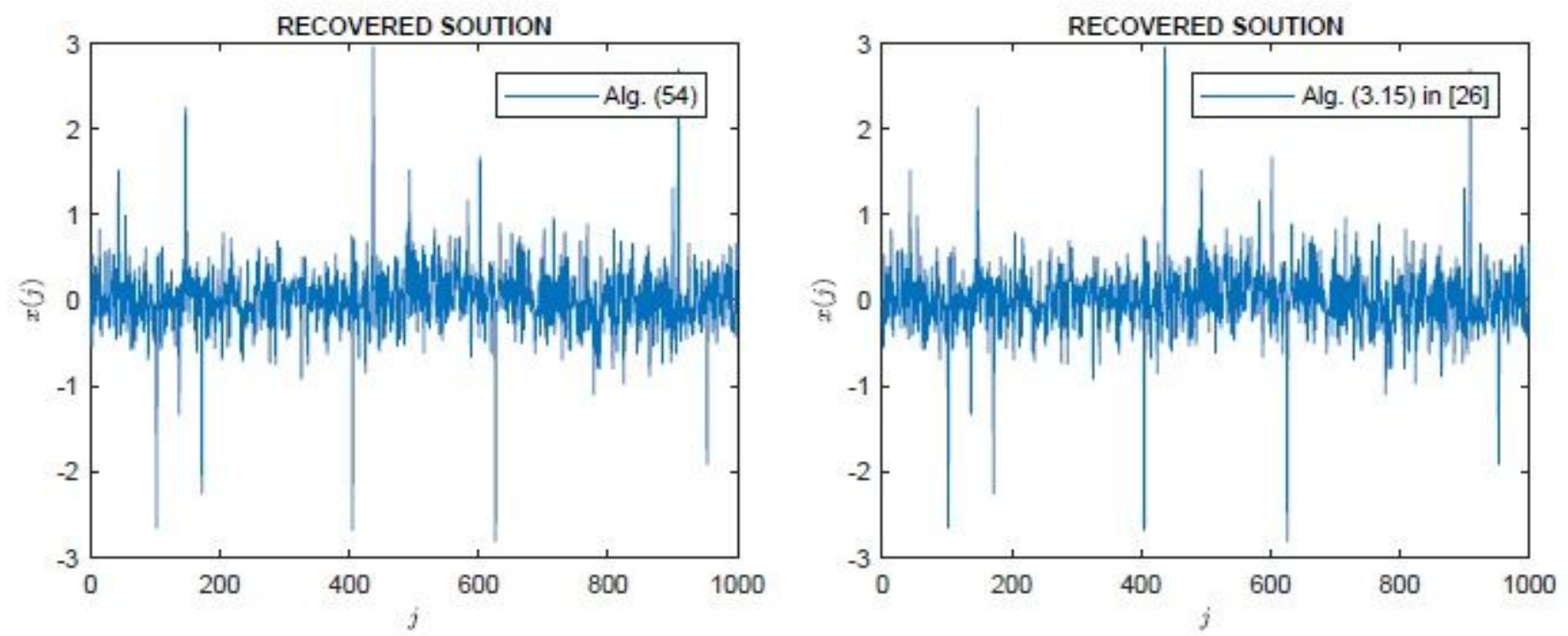

\section{Figure 6}

Recovered signals of Algorithm (54) and Algorithm [26, (3.15)] (qj = 2). 

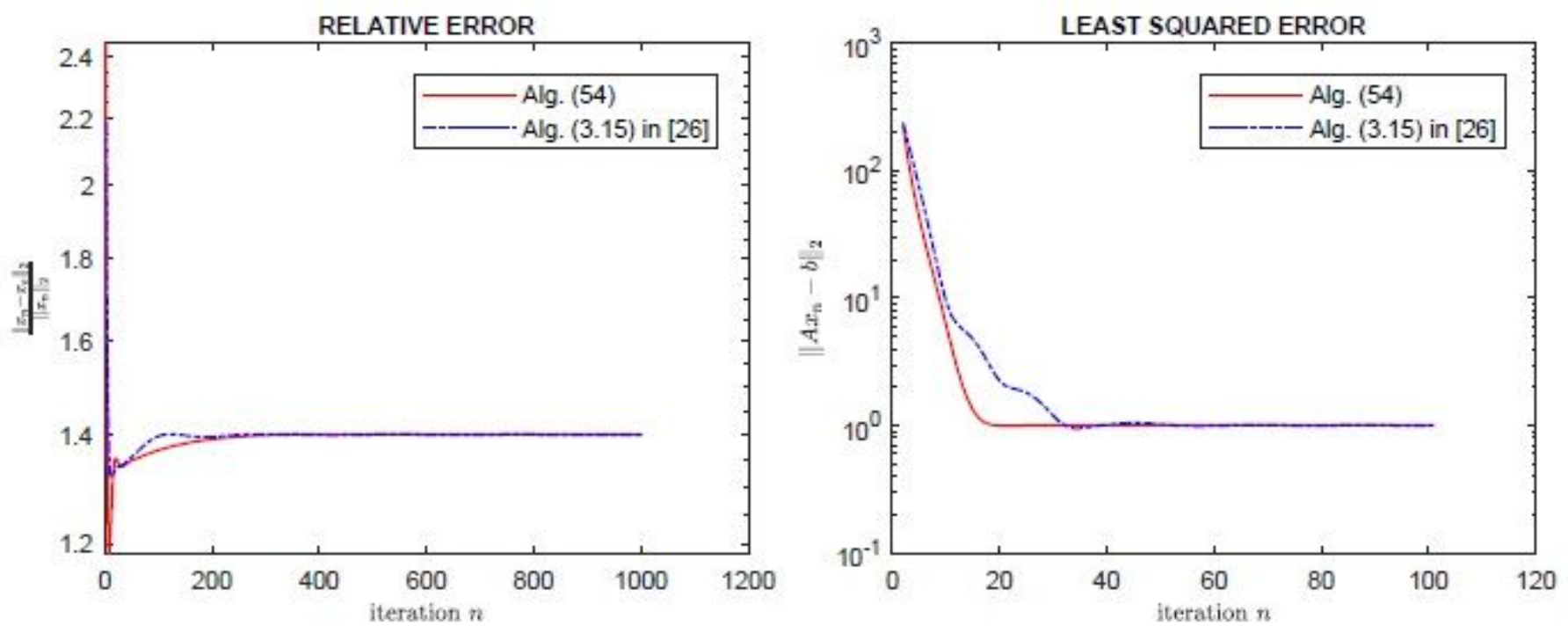

\section{Figure 7}

Relative error and least squared error with Algorithm (54) and Algorithm [26, (3.15)] (qj = 2).
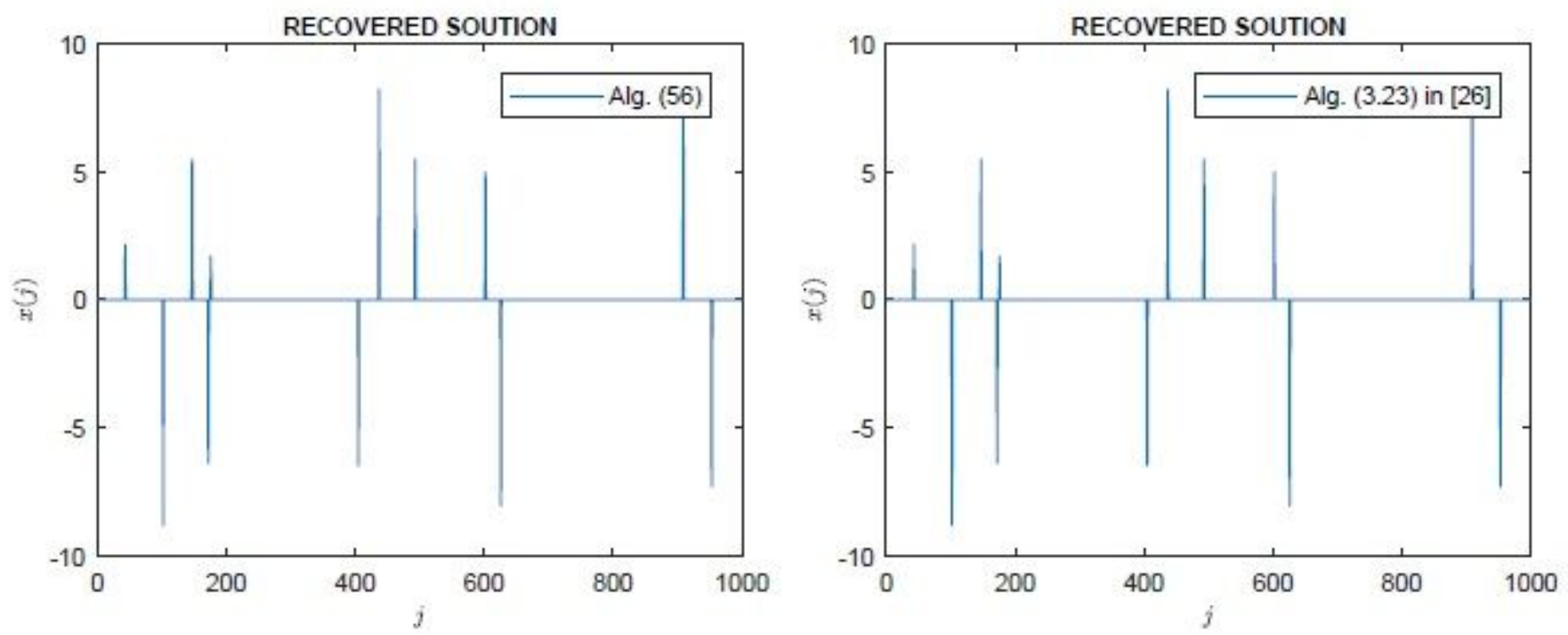

Figure 8

Recovered signals of Algorithm (56) and Algorithm [26, (3.23)] (qj = 1/2). 

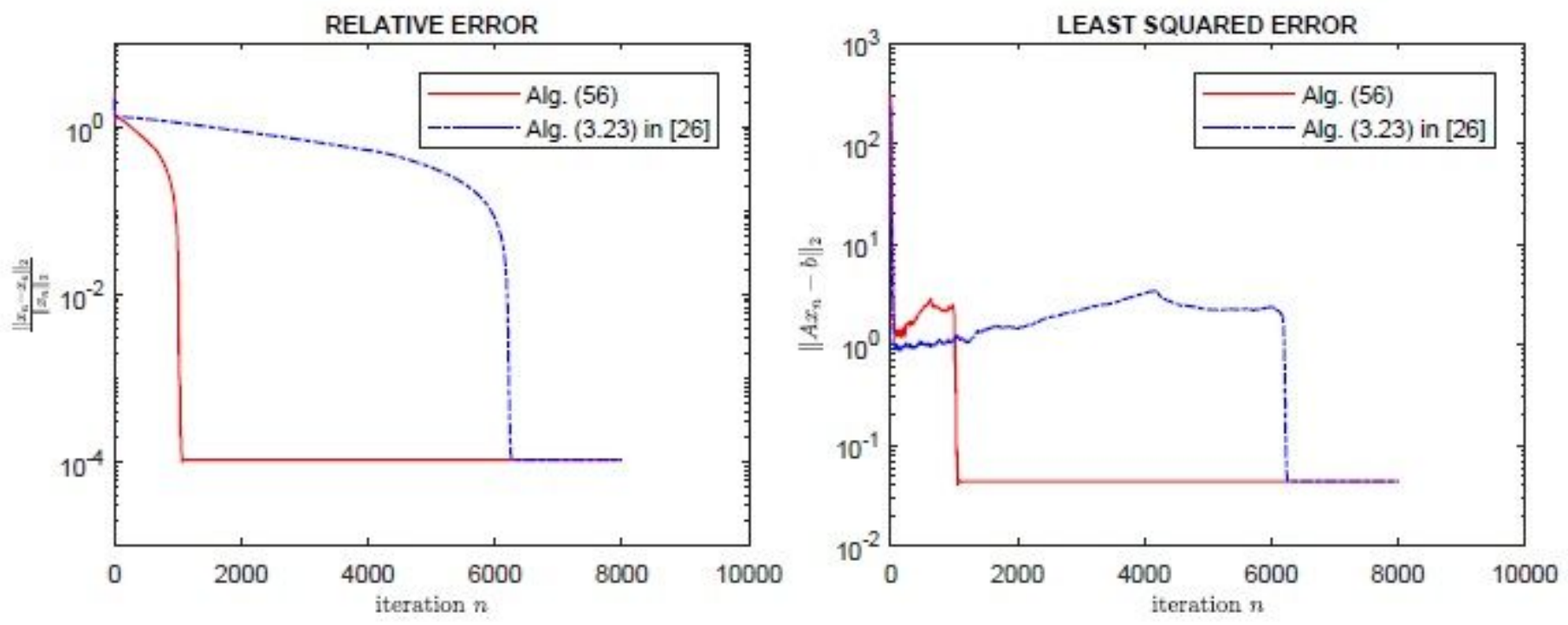

Figure 9

Relative error and least squared error with Algorithm (56) and Algorithm [26, (3.23)] (qj = 1/2 ).
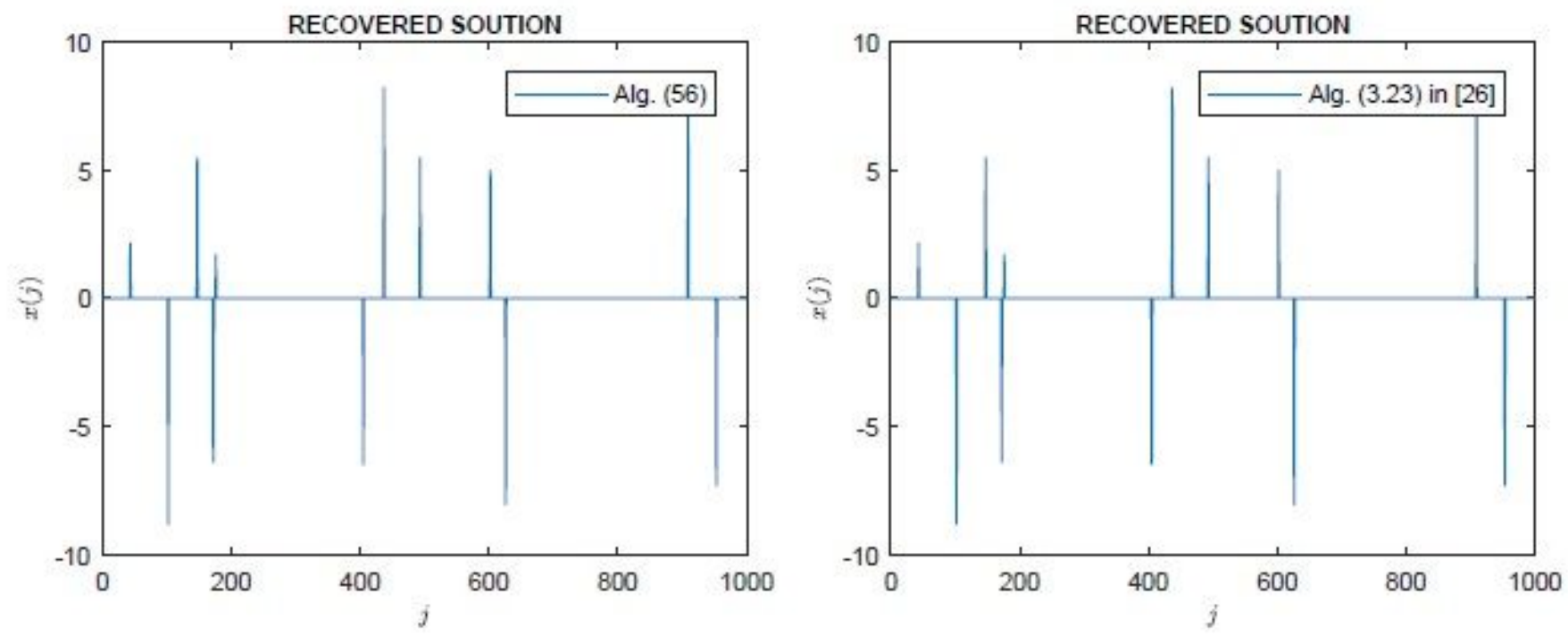

Figure 10

Recovered signals of Algorithm (56) and Algorithm [26, (3.23)] (qj = 2/3). 

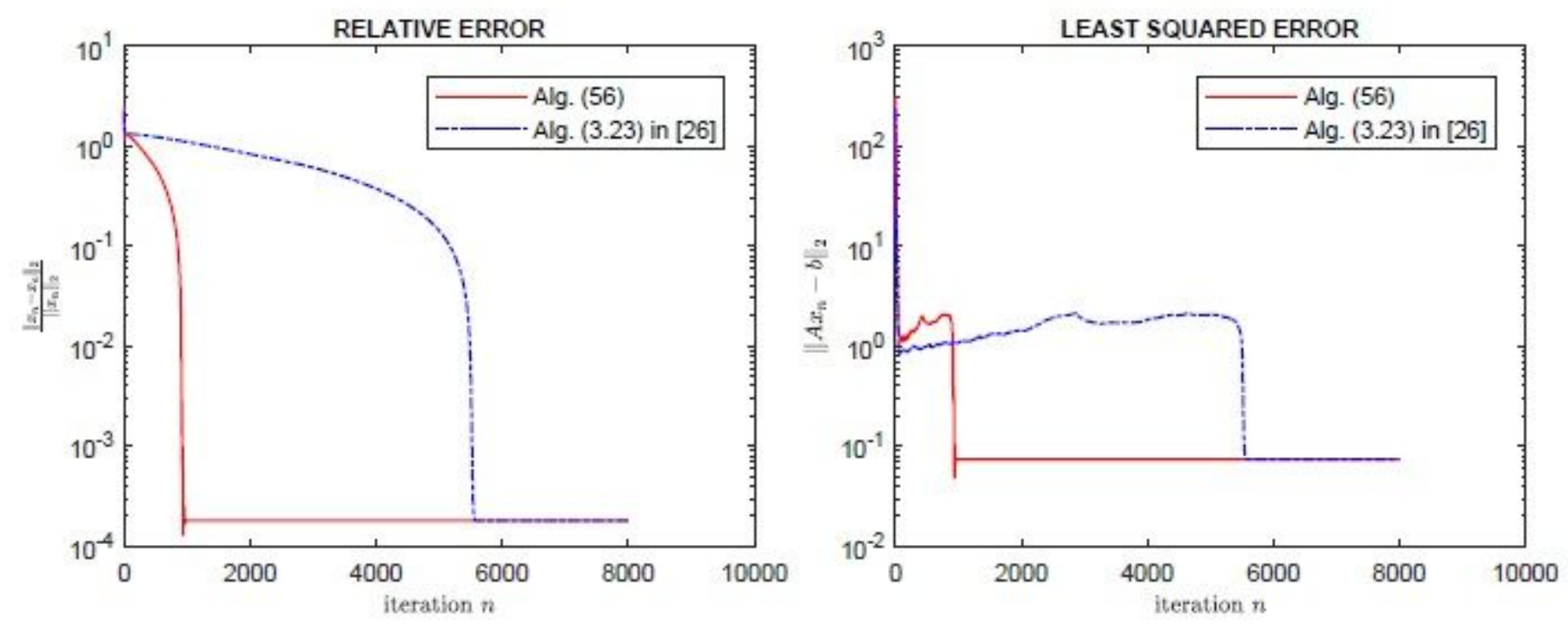

Figure 11

Relative error and least squared error with Algorithm (56) and Algorithm [26, (3.23)] (qj = 2/3 ). 